\title{
REVIEW
}

\section{A review on scaling of earthquake faults}

\author{
Jeen-Hwa Wang *
}

Institute of Earth Sciences, Academia Sinica, Taipei, Taiwan

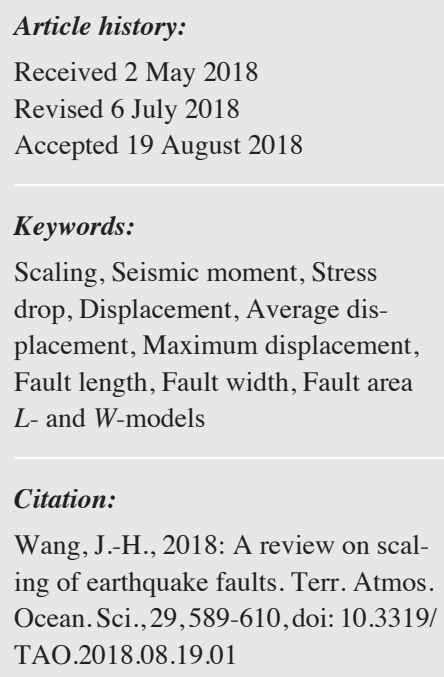

Citation:

Wang, J.-H., 2018: A review on scaling of earthquake faults. Terr. Atmos. Ocean. Sci., 29, 589-610, doi: 10.3319/ TAO.2018.08.19.01

\begin{abstract}
Scaling of earthquake faults is important not only for earthquake physics but also seismic hazard assessment. There have been many studies about this topic. It is significant to compile, review, and discuss those studies. This work reviews the observations and modeling of scaling of fault parameters, including the fault length $(L)$, fault width $(W)$, fault area $(A)$, average displacement $\left(D_{o}\right)$, maximum displacement $\left(D_{\max }\right)$, seismic moment $\left(M_{o}\right)$, and static stress drop $(\Delta \sigma)$. Major issues for reviewing in this work are: (1) assumptions of constant $\Delta \sigma$, of earthquakes and self-similarity of faults; (2) the $L$ - and $W$-models; (3) the $L-D_{o}$ and $L-D_{\max }$ scaling relationships; (4) the $M_{o}-D_{o}$ and $M_{o}-D_{\max }$ scaling relationships; (5) the $D_{o}-A$ scaling relationships; (6) the $M_{o}-A$ scaling relationships; (7) the $L-W$ scaling relationship; (8) the $M_{o}-L$ and $M_{o}-W$ scaling relationships; and (9) modeling of the $M_{o}-L$ scaling based on the onedimensional spring-slider model in the presence of velocity-weakening friction.
\end{abstract}

\section{INTRODUCTION}

Sibson (1984, 1986) and Scholz (1990) assumed that earthquakes occur in the seismogenic zone as displayed in Fig. 1, where $L, W, D_{o}$, and $D(x, t)$ are, respectively, the length, width, average displacement, and displacement on a fault (ruptured) area after an earthquake. These parameters are geometrical properties of earthquake faults. Scaling of the source parameters plays an important role on source physics of earthquakes and seismic hazard assessment. Aki (1967) proposed scaling models to interpret the source spectra of earthquakes, which are dependent on these source parameters, the fault area, $A=L W$, and the maximum displacement on a fault plane, $D_{\max }$. He also assumed that constant static stress drop, $\Delta \sigma$, and self-similarity of earthquakes are physical basis of his model. This leads to the problems of scaling of fault parameters. Aki (1966) defined the seismic moment as $M_{o}=\mu D_{o} L W$ where $\mu$ is the rigidity of the faultzone materials. Obviously, the $M_{o}$ is an important parameter representing the strength of a seismic source and being re-

\footnotetext{
* Corresponding author

E-mail:jhwang@earth.sinica.edu.tw
}

lated to the stress change during faulting. From this definition, naturally seismologists are interested in understanding the scaling relationship between $M_{o}$ and others.

Scaling of earthquake faults has been studied and discussed for a long time (e.g., Aki 1967; Kanamori and Anderson 1975; Scholz 1982; Schwartz and Coppersmith 1984; Shimazaki 1986; Romanowicz 1992; Romanowicz and Rundle 1993; Wang and Ou 1998; Mai and Beroza 2000; Manighetti et al. 2005, 2007; Wesnousky 2006, 2008; Shaw and Wesnousky 2008; Blaser et al. 2010; Kase 2010; Wesnousky and Biasi 2011; Yen and Ma 2011; Carpenter et al. 2012; Stirling et al. 2013; Skarlatoudis et al. 2016; Thingbaijam et al. 2017). Hence, it is one of the important topics of earthquake physics and also useful for seismic hazard estimates. Stirling et al. (2013) compiled a relatively complete dataset of scaling relationships of faults from numerous articles published before 2013. But, some pre-2013 studies were not included in their article.

There have been some studies concerning the relationships between the earthquake magnitude, including moment magnitude $\left(M_{w}\right)$, surface-wave magnitude $\left(M_{s}\right)$, and local magnitude $\left(M_{L}\right)$, and other fault parameters (see Wells and 
Coppersmith 1994; Stirling et al. 2013; and cited references herein; Thingbaijam et al. 2017). Since there is a positive relationship between $M_{o}$ and $M_{w}$ as defined below, related results based on $M_{w}$ are also taken into account by transferring $M_{w}$ into $M_{o}$. However, unlike $M_{w}$ the relationships of $M_{s}$ and $M_{L}$ to $M_{o}$ could be of regional-dependence. Hence, the scaling relationships based on $M_{s}$ and $M_{L}$ are not taken into account hereafter.

Scholz (1982) first pointed out a continued increase in slip with length far beyond the seismogenic depth, i.e., $W$, for large earthquakes. It is necessary to explore this phenomenon. Some authors (e.g., Das 1982; King and Wesnousky 2007; Hillers and Wesnousky 2008; Shaw and Wesnousky 2008) assumed that slip might be penetrating deep below the seismogenic zone during very large ruptures, thus leading to larger $W$. Manighetti et al. (2007) considered that multiple faults can influence the slip-length scaling. Bodin and Brune (1996) reported that large slips occurred in the initiation stage of ruptures might cause longer ruptures. Hanks (1977) suggested that large earthquakes are somehow different than small ones, with a breakdown of constant $\Delta \sigma$ which is observed in small events. Those studies imply in a variety of proposed empirical relations (e.g., Wells and Coppersmith 1994; Hanks and Bakun 2008; WGCEP 2008). These questions have important implications for earthquake physics and for social needs, because the scaling relations are important on seismic hazard estimates (e.g., Wesnousky 2008; WGCEP 2008; Irikura and Miyake 2011).

The previous discussion can lead to a problem with inconsistencies arising in attempts to reproduce empirical ground-motion relations using parameterized kinematic ruptures (Graves et al. 2011). Stirling et al. (2002) proposed a model based on geometrical implications of transition from small circular ruptures to large long rectangular ruptures. They stressed that their model can explain the scaling relationships well: (1) matching well the observed data of
$D_{o}$ versus $L ;(2)$ giving a large crossover length scale matching that observed for magnitude versus length observations (Romanowicz 1992); and (3) giving constant $\Delta \sigma$ scaling for large earthquakes, which matches with that observed for small earthquakes, as well.

Two physical models, i.e., the $L$-model (see Kanamori and Anderson 1975) and the $W$-model (see Scholz 1982), have been proposed to describe scaling of faults. The faults parameters depend on the fault length for the $L$-model and on the fault width for the $W$-model. There have long been debates on the two models.

As mentioned above, many studies about scaling of earthquake faults have been done. It is significant to compile, review, and discuss those studies. This work will review basic concepts, observations, theoretical modeling, and numerical simulations of scaling of geometrical properties of faults. Note that this review study will mainly focus on the scaling exponents of scaling relationships. The issues included are: (1) assumptions of constant $\Delta \sigma$ of earthquakes and self-similarity of faults; (2) the $L$ - and $W$-models; (3) $L-D_{o}$ and $L-D_{\max }$ scaling relationships; (4) $M_{o}-D_{o}$ scaling relationship; (5) $D_{o}-A$ scaling relationships; (6) $M_{o}-A$ scaling relationships; (7) $L-W$ relationship; (8) $M_{o}-L$ and $M_{o}-W$ scaling relationships for small and large earthquakes with various fault types; and (9) modeling of $M_{o}-L$ scaling. The units of fault parameters in use are "bar" for $\Delta \sigma$, "m" for $D_{o}$ and $D_{\text {max }}$, "km" for $L$ and $W$, "km" for $A$, and "dyne-cm" for $M_{o}$. In the followings, the symbols 'SS', 'RE', 'NL', and 'DS' represent, respectively, strike-slip, reverse (or thrust), normal, and dip-slip (including reverse, normal, and oblique). The focal depth is denoted by ' $h$ '.

\section{BASIC CONCEPT}

Figure 1 shows the seismogenic zone (Sibson 1982, 1983; Scholz 1990) and fault (or ruptured) area on a fault

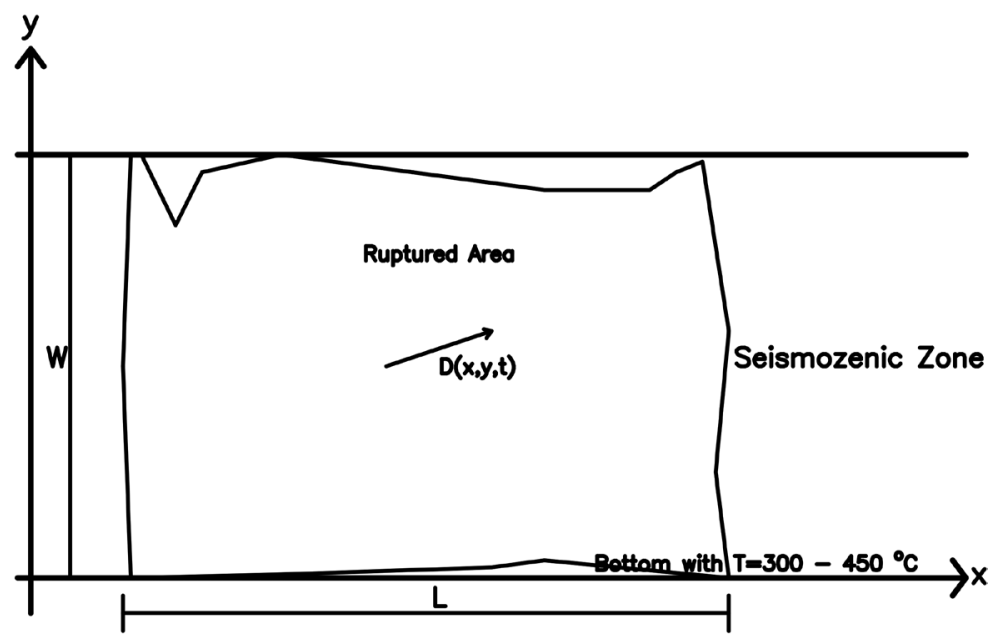

Fig. 1. Seismogenic zone and ruptured area of an earthquake [ $L=$ fault length, $W=$ fault width, and $D(x, y, t)=$ displacement $]$. 
plane during an earthquake and related coordinate system: the fault length along the $x$-axis and the fault width along the $y$-axis. Define $D(\hat{r}, t)$ to be the displacement discontinuity across a fault plane at point $\hat{r}=(x, y, 0)$, where $\hat{r}$ is the position vector, on the fault plane in the rectangular coordinate system and time $t$ in a homogeneous whole space. Since the models used below have an infinite or a very long length, $D(\hat{r}, t)$ can be simplified to be $D(x, t)$ which is the root-meansquared (RMS) average time function of displacements over the fault width, $W$. The bottom of the seismogenic zone with a depth of $W_{s z}$ represents the transition of elasticity to plasticity and is specified with $T=300-450^{\circ} \mathrm{C}$. The value of $W_{s z}$ depends on regional tectonics and thus varies area to area. The fault ruptures at $(0,0,0)$ and stops at $(L, 0,0)$, where $L$ is along the $x$-axis.

When a fault breaks, the static stress changes from an initial value of $\sigma_{0}$ to a final one of $\sigma_{1}$, with a static stress drop of $\Delta \sigma=\sigma_{1}-\sigma_{0}$ and a mean stress of $\sigma=\left(\sigma_{0}+\sigma_{1}\right) / 2$. The static stress drop has the general form (Kanamori and Anderson 1975):

$$
\Delta \sigma=C \mu\left(D_{o} / \Lambda\right)
$$

where $D_{o}$ and $\Lambda$ denote, respectively, the average displacement and linear dimension (either $L$ or $W$ ) of a fault plane. The ratio $D_{o} / \Lambda$ denotes the strain change or strain drop. The constant $C$ depends on the fault type, for example, $C$ $=7 \pi / 16$ for a circular fault (Scholz 1990). This gives $\Delta \sigma=$ $(7 \pi / 16) \mu\left(D_{o} / R\right)$ where $\Lambda$ is the radius of the circular fault, $R$. From $M_{o}=\mu D_{o} L W=\mu D_{o} A$, we have $M_{o}=\left(16 \Delta \sigma / 7 \pi^{3 / 2}\right)$ $A^{3 / 2}$, thus giving

$$
\log \left(M_{o}\right)=1.5 \log (A)+\log \left(16 \Delta \sigma / 7 \pi^{3 / 2}\right)
$$

From the observations, Kanamori and Anderson (1975) suggested $M_{s} \sim \log (A)$ for most of earthquakes. This leads to $\log \left(M_{o}\right)=1.5 M_{s}+\log \left(16 \Delta \sigma / 7 \pi^{3 / 2}\right)$. The observations obtained by Kanamori and Anderson (1975) and Hanks (1977) show average $\Delta \sigma \approx 100$ bars for most of earthquakes. This gives

$\log \left(M_{o}\right)=1.5\left(M_{s}+10.7\right)$

From Eq. (3), Hanks and Kanamori (1979) defined the moment magnitude as

$M_{w}=(2 / 3) \log \left(M_{o}\right)-10.7$

Anderson et al. (1996) addressed a problem if the slip rate on a fault, $\gamma_{F}$, can be a factor in influencing estimates of $M_{w}$ for shallow continental surface rupturing earthquakes. Based on 43 earthquakes from the database of Wells and
Coppersmith (1994), they suggested that the estimate of $M_{w}$ from $L$ can be improved by incorporating $\gamma_{F}$ based on the formulae: $M_{w}=5.12+1.16 \log (L)-0.20 \log \left(\gamma_{F}\right)$. However, this formulae exhibits that the influence on $M_{w}$ is smaller due to $\gamma_{F}$ is smaller due to $L$, because the related coefficient $(=0.20)$ is smaller than that $(=1.16)$ for $\log (L)$.

Note that the physical basic concept as mentioned above is related to the nine scaling relationships of earthquake faults reviewed below.

\section{MEASURES OF FAULT PARAMETERS AND INFERENCE OF SCALING LAWS}

Wells and Coppersmith (1994) compiled a relatively complete data of source parameters of earthquake faults. Wells and Coppersmith (1994) gave detailed description about the measurement and uncertainty of the surface and subsurface fault parameters. Here, only a simple description is given. Essentially, there are two kinds of fault length: one is surface rupture length, $L_{o}$, and the other sub-surface rupture length, $L_{s}$. When surface ruptures occur after the occurrence of a mainshock, $L_{o}$ can be measured directly from field surveys. The reasons to cause uncertainty in measuring $L_{o}$ comes are: (1) incomplete field surveys and studies of the ruptured fault zone; (2) a lack of outcrops at some localities; (3) different interpretations of the properties and amount of surface ruptures and deformations by different researchers; and (4) different reported values of fault lengths and surface displacements by different field workers. For the sub-surface rupture length, $L_{s}$, and fault area, $A$, there are two commonly-used ways to evaluate the two parameters. The first way is based on the spatial pattern of early aftershocks (Kanamori and Anderson 1975; Dietz and Ellsworth 1990). Because the aftershocks may expand outwards from the source area of the mainshock, only the zone of aftershocks that occur within a few hours to a few days after the mainshock and also follow the Omori law can be the representative of the co-seismic ruptured area. The reasons to cause uncertainties for measuring subsurface rupture parameters are: (1) hypocentral accuracy of aftershocks; (2) identification of the strike and dip of the rupture plane from aftershocks; (3) identification of the length and downdip width from the spatial distribution of aftershocks; and (4) estimates of the respective lengths of multiple earthquake rupture sequences. In addition, since many aftershocks occur on the sub-faults rather than on the main fault, the fault area estimated from the aftershock area should be larger than the real one. From 53 earthquakes having both $L_{o}$ and $L_{s}$, Wells and Coppersmith (1994) found that $L_{o}$ is, on the average, $\sim 75 \%$ of $L_{s}$ and $L_{o} / L_{s}$ increases with $M_{w}$.

The second way is based on the finite-fault slip model inversed from seismograms and geodetic data (e.g., Yen and Ma 2011; Thingbaijam et al. 2017). This way will rely on the reliability of geodetic and seismologic modeling, including 
data quality and inversion technique. Yen and Ma (2011) took the effective fault length $\left(L_{e}\right)$, effective fault width $\left(W_{e}\right)$, effective fault area $\left(A_{e}=L_{e} W_{e}\right)$, and effective average displacement $\left(D_{o e}\right)$ which are estimated using the autocorrelation technique (see Bracewell 1986) for the source slip function. Of course, the reliability of inversed slip function and the weighting factor used in autocorrelation can influence the estimated results. In general, $L_{e}$ is shorter than $L_{s}$. Ichinose et al. (2006) used the combined area of asperities, $A_{a}$ (in $\mathrm{km}^{2}$ ), which is usually smaller than the real fault area. From the slip models of plate-boundary earthquakes in the Japan inferred from strong-motion, teleseismic, geodetic, or tsunami records, Murotani et al. (2008) found the exponents of the scaling relationship $M_{o}-A, M_{o}-D_{o}$, and $M_{o}-A_{a}$ are 1.4, 0.4, and 1.2 times larger, respectively, than those of crustal earthquakes. On the other hand, the ratios $A_{a} / A_{a}$ and $D_{o a} / D_{o}$ for plate boundary earthquakes are the same as those for crustal earthquakes, and thus they concluded that plate-boundary and crustal earthquakes share similar source characteristics.

The values of $D_{o}$ and $D_{\max }$ are usually obtained directly from field surveys and finite-fault slip models inversed from seismograms and geodetic data. Since $D_{o}$ is the average of displacements over the fault and $D_{\max }$ is the largest displacement on a fault plane, $D_{o} / D_{\max }$ should be smaller than 1 with a maximum of 1 . The displacements over the fault plane should be somewhat uniform when $D_{o} / D_{\max } \approx 1$ and non-uniform over the fault plane when $D_{o} / D_{\max }<1$. Unlike surface rupture length, $D_{o}$ and $D_{\max }$ for many earthquakes often are poorly reported. The most common measures of displacements are the maximum horizontal and/or vertical surface displacement. For some earthquakes, the field surveys were made weeks to years after the event, the measured values could include afterslip, slip caused by aftershocks, and fault creep. The surface ruptures usually vary point to point and the long displacements occur in a limited segment of the fault zone. The value of $D_{o}$ is commonly calculated from multiple measurements of displacements along the fault zone. When the number of multiple measurements is small, the calculated value of $D_{o}$ is unlikely the true one. From field data, Wells and Coppersmith (1994) observed $D_{o} / D_{\max }=0.2-0.8$, with an average of $D_{o} \approx 0.5 D_{\max }$. They also reported that $D_{o} /$ $D_{\max }$ does not vary systematically with $M_{w}$, especially for earthquakes with $D_{o}$ which was estimated from $M_{o}$ obtained from the finite-fault slip models. They also studied the ratio of average subsurface displacement, $D_{o s}$, and the maximum subsurface displacement, $D_{\max s}$. The gained $D_{o s} / D_{\max s}=0.14$ - 7.5 and $D_{o s} / D_{o}=0.25$ - 6.0. Meanwhile, the two ratios are not dependent on $M_{w}$. From 44 earthquakes having the values of both $D_{o s}$ and $D_{\text {maxs }}$, they found $D_{o s} / D_{\operatorname{maxs}}=0.76$. This indicates that for most earthquakes $D_{o s}$ is shorter than $D_{\max s}$. From 32 earthquakes having the values of both $D_{o s}$ and $D_{o}$, they found $D_{o s} / D_{o}=1.32$. Hence, they concluded that $D_{o s}$ is longer than $D_{o}$ and shorter than $D_{\max }$. This indicates that for these earthquakes the displacements happen at shallow depth on the fault plane.

The reasons to cause uncertainties in the displacements are: (1) incomplete or insufficient measures of displacements along the fault trace due to a lack of suitable features (e.g., stratigraphy, streams, or cultural features) for conducting measurements; (2) distribution of displacement along multiple fault strands or distributed shearing over a broad fault zone; (3) changes of the fault scarp due to landsliding or erosion; (4) an increase in slip due to afterslip, aftershocks, and post-event creep; (5) inaccurate locations of conducting slip measures; and (6) the measurements being mixed up by slip caused by historical or paleo-earthquakes.

The value of $M_{o}$ is commonly obtained from the finite fault slip model (see Aki and Richards 1980). For some events, the seismic moment may also be evaluated from geological observations with $L$ and $D_{o}$ from field measures of offsets along the outcrops of surface trace of an earthquake fault (cf. Wesnousky 2008). He denoted this type of seismic moment as $M_{o}{ }^{G}$. Of course, the measures can be done only for the earthquakes which are large enough to break the ground surface and their values of rupture widths can be independently estimated from the finite-fault slip model. He stressed that there is a good linear correlation between $\log \left(M_{o}{ }^{G}\right)$ and $\log \left(M_{o}\right)$ and the values of $M_{o}{ }^{G}$ usually fall within the range of estimated values of $M_{o}$ for larger events.

There is long historical development about the measures of source parameters and studies of scaling laws of earthquake faults. A simple description is given below. From 1980's to 2000's, almost all of the papers concerning scaling of source parameters of earthquake faults were completed based on continental strike-slip earthquakes. Meanwhile, much of the literature focused on the debate, for example, the debate between Scholz et al. (1986) and Romanowicz (1992), over scaling relationships of $W$ versus $L$ was also on strike-slip earthquakes. Of course, Wells and Coppersmith (1994) could be the most notable exception in this time period. They compiled a relatively complete data set of source parameters. In their data set, there are 389 world-wide earthquakes with $M_{w}=4.2-8.5$ occurred before 1994, including 305 instrumentally-determined events (post-1900) and 84 pre-instrumental events (pre-1900). The pre-instrumental earthquakes include events from A.D. 701 and those inferred from paleoseismic data. From those data, Stirling et al. (2002) found the estimates of $D_{o}$ and $M_{w}$ for crustal earthquakes in the pre-instrumental era (pre-1900) are greater than the estimates calculated from the scaling relationships inferred from instrumental data. After 1990's, the scaling relationships of world-wide events with other types of faulting were published (e.g., Blaser et al. 2010) as well as regionally specific ones, for examples, Stirling et al. $(2002,2008,2013)$ for New Zealand and Yen and Ma (2011) for Taiwan. Since 2010's, more general scaling relationships were developed (e.g., Leonard 2010, 2014; Thingbaijam and Mai 2016; Thingbaijam et al. 2017) by using 
expanded data sets and new methods.

In order to study the scaling law between two source parameters, it is common to infer the regression relationship between them. The regression equation for two variables $x$ and $y$ usually has the following form: $\log (y)=a+b \times \log (x)$, thus leading to the scaling law: $y \sim x^{b}$. Usually, the standard errors, i.e., $\delta a$ for $a$ and $\delta b$ for $b$, should be included in the regression equation, i.e., $y=(a \pm \delta a)+(b \pm \delta b) x$. Hence, the value of $\delta b$ must be included in the following scaling law: $y \sim x^{b \pm \delta b}$. In addition, the standard deviation (denoted by $S D$ hereafter) of the regression equation is also estimated by the researchers.

However, there are four problems for the present study. The first problem is that a few source materials did not provide the values of $\delta b$ and/or $S D$. The third problem is that for some source materials, the $\delta b$ values was evaluated for the $x \sim y^{b^{\prime} \pm \delta b^{\prime}}$, and then this scaling law must be conversed to $y \sim x^{b \pm \delta b}$. It is questionable to estimate $b$ and $\delta b$ from $b$ ' and $\delta b^{\prime}$, respectively. It is possible to obtain $b=1 / b$ ' and the $\delta b$ value when the major-axis method (York 1967) or the general orthogonal least-squared method (e.g., Thingbaijam et al. 2017) are taken for regression. However, numerous authors used the ordinary method (for instance, Wells and Coppersmith 1994). There is no problem when the scaling law $y \sim x^{b \pm \delta b}$ is inferred from the data. Whereas, it is difficult to gain the reliable values of $b$ and $\delta b$, when the authors only estimated the values of $b^{\prime}$ and $\delta b^{\prime}$. In addition, for some source materials the regression equations were estimated based om $M_{w}$ rather than on $M_{o}$, and thus the regression equation is: $M_{w}=(a \pm \delta a)+(b \pm \delta b) \log (x)$ or $\log (x)=$ $\left(a^{\prime} \pm \delta a^{\prime}\right)+\left(b^{\prime} \pm \delta b^{\prime}\right) M_{w}$. It is necessary to converse such a regression equation into $M_{o} \sim x^{n}$ where $n=2 b / 3$ by using Eq. (4), i.e., $M_{w}=(2 / 3) \log \left(M_{o}\right)$ - 10.7. Hence, it is impossible to gain the reliable values of $\delta b$ and/or $S D$. Anyway, the values of $\delta b$ and/or $S D$ will be included in the respective resultant scaling law when the two values have been estimated by the original authors. However, the two values will be not listed the tables mentioned below, because of complexity of the two values obtained from different source materials.

In addition, from the relatively complete data set provided by Wells and Coppersmith (1994) we can see that the numbers of earthquakes decrease from the strike-slip (SS) events, reversed (RE) events, to normal (NL) events. Hence, the uncertainty of scaling exponent could increases in the opposite direction. Note that some authors combined the data of RE, NL, and oblique events together to form a data set for the dip-slip (DS) events and some others took all slip-type (including SS, RE, and NL) events together to study scaling laws.

\section{CONSTANT $\Delta \Sigma$ OF EARTHQUAKES AND SELF-SIMILARITY OF FAULTS}

In order to build up the scaling model of seismic spec- tra, Aki (1967) assumed that large and small earthquakes are similar geometrically. This leads to the so-called self-similarity of earthquakes and can be seen that the curves shown in each panel show a similar shape. This means that for geometrically similar earthquakes $W$ is proportional to $L$; and for physically similar events all the non-dimensional quantities obtained from the source parameters should be the same. Thus, $D_{o}$ is proportional to $L$ as well as $W$. This implies that for an earthquake with Starr fracture (Starr 1928), the preexisting stress or strength is constant and independent of source size. This point was first assumed by Tsuboi (1956). Aki (1967) also assumed that if earthquakes are geometrically similar, $\Delta \sigma$ is constant and independent of earthquake magnitude (denoted by $M_{w}$ hereafter). This leads to $D_{o} \sim L$ [from Eq. (1)], which yields constant $\Delta \sigma$ for all earthquakes. Nevertheless, Aki (1967) gave an example of departure from the assumption of self-similarity and constant $\Delta \sigma$.

There are debates for the problem of constant $\Delta \sigma$. Some observations (e.g., Aki 1972; Kanamori and Anderson 1975; Hanks 1977; Boore and Atkinson 1987; Somerville et al. 1987; Heimpel and Malin 1998; Bilek and Lay 1999; Imanishi and Ellsworth 2006; Shaw 2009) show that earthquakes are scale invariant with $\Delta \sigma \approx 100$ bars being independent of $M_{w}$. The log-log plot of $M_{o}$ versus $L$ obtained by Hanks (1977) shows constant $\Delta \sigma$ in a large range of $M_{o}$ and thus it is the strongest evidence of self-similarity of earthquakes. From 41 moderate and large earthquakes, Geller (1976) inferred the rise time of faulting: $t_{R}=16 A^{1 / 2} / 7 \pi^{3 / 2} \beta$ based on the assumptions of constant effective stress, $\sigma_{e}$, and $\Delta \sigma$. Due to $W=L / 2$, he got $t_{R} \sim L$. These observations combine to confirm the $L$-model for which $M_{o}$ scales with $L^{2}$.

On the other hand, Nuttli (1983a, b) observed constant $\Delta \sigma$ for plate margin earthquakes, but an increase in $\Delta \sigma$ with $M_{o}{ }^{1 / 4}$ for mid-plate events. Some studies (e.g., Wells and Coppersmith 1994; Hanks and Bakun 2008; WGCEP 2008) observed an increase in $\Delta \sigma$ with $M_{w}$ for large earthquakes. Allmann and Shearer (2009) observed higher $\Delta \sigma$ for SS earthquakes than for $\mathrm{N}$ and R events. Kanamori et al. (1990, 1993) and Ma and Kanamori (1994) observed abnormally high $\Delta \sigma$ for the 1988 Pasadena earthquake and the $1991 \mathrm{Si}-$ erra Madre earthquake. They suggested that these high $\Delta \sigma$ events occurred near the base of the seismogenic zone, thus indicating that these fault systems can support high stress that will be released in the main events. Nadeau and Johnson (1998) observed that the values of $\Delta \sigma$ were high in the Parkfield repeating events and showed an inverse relation to $M_{w}$. Several authors (Archuleta 1982; Fletcher 1982; Haar et al. 1984; Fletcher et al. 1986) reported a significant difference in $\Delta \sigma$ between large and small events. Bizzarri (2011) found the failure of constant $\Delta \sigma$. From the near-fault seismograms of the $1999 M_{s} 7.6$ Chi-Chi, Taiwan, earthquake, Huang et al. (2001) and Hwang et al. (2001) observed higher $\Delta \sigma$ on the northern fault plane with two-degree-of-freedom ruptures than on the southern plane with one-degree-of-freedom 
ruptures. Manighetti et al. (2007) assumed the variability of large $\Delta \sigma$ of earthquakes. For large Taiwan's earthquakes, Yen and Ma (2011) observed $\Delta \sigma \approx 10-100$ bars for most of events with $M_{o}>10^{24}-10^{25}$ dyne-cm and higher for those with $M_{o}<10^{24}-10^{25}$ dyne-cm, thus suggesting non-constant $\Delta \sigma$. Hardebeck and Aron (2009) found that high $\Delta \sigma$ occurs in a deep cluster of RE earthquakes. Several authors (Asano et al. 2003; Allmann and Shearer 2009; Hardebeck and Aron 2009) reported the dependence of $\Delta \sigma$ on depth. Depth-dependence of $\Delta \sigma$ also indicates failure of constant $\Delta \sigma$. On the other hand, Yen and Ma (2011) did not observe dependence of large $\Delta \sigma$ on depth and fault type. From 5 inland crustal earthquakes with $M_{w}=5.6-6.9$ and depths ranging from 7.8 - $14.1 \mathrm{~km}$ in Japan during 2004 - 2008, Asano and Iwata (2011) derived their empirical relationship and found an increase $\Delta \sigma$ with depth. From 8 earthquakes with $M_{L}=3.8$ - 6.8 and depths ranging from $8.31-25.22 \mathrm{~km}$ in the Nantou area of Taiwan during 1999 - 2013, Wen et al. (2017) revealed an increase in $\Delta \sigma$ with depth.

There are debates for the problem of self-similarity of earthquakes. Some authors (e.g., Hanks 1977; Romanowicz 1992; Scholz 1994a, 1997; Wang and Ou 1998; Mai and Beroza 2000; Prieto et al. 2004; Shaw and Wesnousky 2008) have suggested that both self-similarity and quasi$L$ model scaling could be maintained if slip in the largest earthquakes penetrates beneath the brittle-ductile transition, i.e., the width of the seismogenic zone.

For the intraplate earthquakes having higher $\Delta \sigma$ (Kanamori and Anderson 1975) and those with long recurrence intervals (Kanamori and Allen 1986), self-similarity fails. Romanowicz (1992) observed that large earthquakes are not similar to small events. Mai and Beroza (2000) found that self-similarity exists for DS events, yet not for large SS ones. In addition, several authors (Hartzell and Heaton 1988; Funning et al. 2013; Denolle and Shearer 2016) observed non-self-similarity.

\section{5. $L$ - AND $W$-MODELS}

There is an interesting, important problem: How do fault parameters, such as $L, W, A, D_{o}, v_{R}$ (rupture velocity), $T_{D}$ (duration of rupture) etc., scale one to another? Based on the scaling model proposed by Aki (1967), Kanamori and Anderson (1975) assumed the following similarity relations: (1) $W / L=c_{1}=$ constant (aspect ratio for geometrical similarity); (2) $D_{o} / L=c_{2}=$ constant (constant static stress drop); and (3) $v_{R} T_{D} / L=c_{3}=$ constant (dynamics similarity). Their assumptions are based on the fault length and thus represent an $L$-model for fault scaling. Accordingly, the relations between seismic moment and fault parameter have the following basic forms: $M_{o} \sim L^{3}$ to correlate $M_{o}$ and $L, M_{o} \sim W^{3}$ to correlate $M_{o}$ and $W, M_{o} \sim D_{o}{ }^{3}$ to correlate $M_{o}$ and $D_{o} ;$ and $M_{o} \sim A^{1.5}$ to correlate $M_{o}$ and $A$.

According to Eq. (1), related parameters are $\Lambda=W / 2$ and $C=2 / \pi$ infinite length strike-slip ruptures and $C=4(\lambda$ $+\mu) / \pi(\lambda+2 \mu)$, where $\lambda$ and $\mu$ are the Lamé constants of elastic materials, for dip-slip ruptures of width $W$ in a whole space (Starr 1928; Knopoff 1958). Obviously, $\Delta \sigma$ and $M_{o}$ both scale only with $W$. This presents the $W$-model (see Scholz 1982). This model means that large events with the same fault width have the same average displacement in spite of the fault length. Meanwhile, for the $W$-model $t_{R}$ is related to $W$ for constant $v_{R}$. The $L$ - and $W$-models are two end-member models for fault scaling. A $W$-model implies constant $\Delta \sigma$ for all earthquakes in the same tectonic setting, and therefore that earthquakes are self-similar. The $L$-model does not need to imply self-similarity, but is consistent with the idea that large earthquakes (i.e., earthquakes that rupture the full thickness of the brittle upper crust) grow by increasing their rupture length, i.e., $D_{o} \sim L$. There has been a longstanding debate concerning the two models.

Numerous studies (Geller 1976; Scholz 1982, 1994a; Bodin and Brune 1996; Pegler and Das 1996; Wang 1997; Wang and Ou 1998; Mai and Beroza 2000; Shaw and Scholz 2001; Thingbaijam et al. 2017) prefer the $L$-model. On the other hand, Romanowicz (1992) and Romanowicz and Rundle (1993) observed a linear relation between $L$ and $M_{o}$, implying approximately constant average slip on the order of $3-5 \mathrm{~m}$ for the largest SS earthquakes. This observation supports the $W$-model for which $D_{o}$ is governed by $W$. However, her approach was different from that of Scholz (1982) in which $W$ was allowed to vary.

It is significant to explore the possible dependence of fault scaling on $A$ in the seismogenic zone (see Scholz 1990) as briefly displayed in Fig. 1. An event is a characteristic earthquake (Schwartz and Coppersmith 1984) when $L=W$, in which $L=L_{\mathrm{c}}$ and $W=W_{s z}$ are, respectively, the length and width of the seismogenic zone as displayed in Fig. 2. Its ruptured area is $A_{c}=L_{\mathrm{c}} W_{s z}=W_{s z}^{2}$ and called the characteristic area hereafter. For an event, its ruptures with $A<$ $A_{c}$ can grow along both the length- and width-directions as displayed by the left-hand-side small square in Fig. 2 when it has two degrees of freedom. In other word, $L$ and $W$ can both increase with earthquake size. This gives $W \sim L$ and thus $A \sim L^{2}$ or $A \sim W^{2}$. Obviously, the events with $A<A_{c}$ can be similar one to another. For an event, its ruptures with $A>$ $A_{c}$ can grow only along the length-direction as displayed by the right-hand-side small square in Fig. 2. In other word, $L$ increases and $W$ retains constant. This exhibits one-degreeof-freedom ruptures and thus gives $A \sim L$ and the events with $A>A_{c}$ can also be similar one to another. But, the event with $A<A_{c}$ cannot be similar to that with $A>A_{c}$. Consequently, the characteristic earthquake separates scaling of faults into two parts. Hence, the $L$-model is operative for $A>A_{c}$ or $L>$ $W_{s z}$; while either the $L$-model or the $W$-model can be applied to describe the source behavior for $A<A_{c}$ or $L<W_{s z}$.

Based on static dislocation theory with constrain that slip is confined to the seismogenic layer, $\Delta \sigma$ increases with 
$L$ for large earthquakes. This implies that large earthquakes differ from small ones. Shaw and Wesnousky (2008) used a 3-D elastodynamic model to show that an increase in $\Delta \sigma$ with $L$ may be satisfied while maintaining constant $\Delta \sigma$ across the entire spectrum of earthquake sizes when slip is allowed to penetrate below the seismogenic zone into an underlying zone specified with velocity-strengthening friction.

\section{6. $L-D_{o}$ AND $L-D_{\text {MAX }}$ SCALING RELATIONSHIPS}

\subsection{Observations}

Based on the assumption of constant $\Delta \sigma, D_{o}$ linearly increases with $L$. Hence, $L-D_{o}$ and $L-D_{\max }$ scaling relationships are first reviewed. Scholz and Cowie (1990) observed a linear correlation between $\log \left(D_{o}\right)$ and $\log (L)$, thus implying a power-law relationship between $D_{o}$ and $L$. For 244 world-wide shallow earthquakes with $M_{w}=4.7-8.2$, Wells and Coppersmith (1994) observed $D_{o} \sim L^{1.04 \pm 0.13}$ (with $S D=$ 0.32 ) and $D_{\max } \sim L^{1.16 \pm 0.09}$ (with $S D=0.36$ ) for SS events, $D_{o} \sim L^{0.31 \pm 0.27}$ (with $S D=0.40$ ) and $D_{\max } \sim L^{0.42 \pm 0.23}$ (with $S D$ $=0.43$ ) for RE events, $D_{o} \sim L^{1.24 \pm 0.49}$ (with $S D=0.37$ ) and $D_{\max } \sim L^{1.51 \pm 0.35}$ (with $S D=0.41$ ) for NL events, $D_{o} \sim L^{0.88 \pm 0.11}$ (with $S D=0.36$ ) and $D_{\max } \sim L^{1.02 \pm 0.09}$ (with $S D=0.41$ ) for all slip-type events based on the surface rupture length. Obviously, the scaling exponent is the largest for NL event, the second largest for $\mathrm{SS}$ events and the smallest for the RE events. From 27 data of $D_{o}$ and $L$ (18 plate-boundary earthquakes and 9 away from transform plate boundaries), Bodin and Brune (1996) observed a power-law increase of $D_{o}$ with $L$. From the world-wide earthquakes, Wang and Ou (1998) observed $D_{o} \sim L^{1.03 \pm 0.26}$ based on the surface rupture length and $D_{o} \sim L^{1.01 \pm 0.18}$ based on the subsurface rupture length for all slip-type events. Basically, the scaling of $D_{o}$ versus $L$ is almost the same for the two types of rupture length.

From the finite-fault slip models of 18 earthquakes $(8$
SS and 10 DS events), for the source data from the finitefault rupture models of 31 world-wide earthquakes with $M_{o}$ $=2.2 \times 10^{17}-1.2 \times 10^{21} \mathrm{NM}$, Mai and Beroza (2000) obtained $D_{o} \sim L^{1.82 \pm 0.46}$ (with $S D=0.34$ ) for $8 \mathrm{SS}$ events; $D_{o} \sim L^{0.52 \pm 0.26}$ (with $S D=0.36$ ) for $11 \mathrm{DS}$ events; and $D_{o} \sim L^{0.65 \pm 022}$ (with $S D=0.35$ ) for 31 all slip-type events. Obviously, Mai and Beroza (2000) observed that $D_{o}$ nonlinearly increases with $L$ with a decrease in the increasing rate of $D_{o}$ with $L$, especially for large SS events. Their observation suggests that the finite fault width strongly influences the displacement for very large SS earthquakes. From the log-log plot of $D_{o}$ versus $L$ for large crustal earthquakes compiled by Scholz (1994b), Shaw and Scholz (2001) observed $D_{o}$ increases nonlinearly with $L$ and the increasing rate decreases with increasing $L$.

For numerous $M_{w} \geq 6$ earthquakes, Manighetti et al. (2007) analyzed the $L-D_{o}$ relationship and also proposed a new $D_{\max }-L$ relationship based on the concept of multiple segment-ruptures. The number of main fault segments depends on the strength of the inter-segment zones, and the strength is influenced by the structural maturity of the faults. They assumed that each broken segment can be roughly scaled as a crack, while the total multi-segment rupture cannot. The value of $\Delta \sigma$ on an individual segment is roughly constant, varying between 3.5 to $9 M P a$. The $\Delta \sigma$ is lower for more mature faults than for immature ones.

King and Wesnousky (2007) and Wesnousky (2008) observed that the linear relationships between $D_{o}$ and $L$ and those between $D_{\max }$ and $L$ are stronger for the DS events than for the SS ones. Different linear curves must be applied separately to various slip types of faults. The data points for RE and NL events can be well fitted by a straight line, yet not for SS events. They further considered log-linear function: $D_{o}=a+b \log (L)$ and power-law function: $D_{o}=b L^{d}$ for $\mathrm{SS}$ events. The log-linear fit is formulated to constrain the

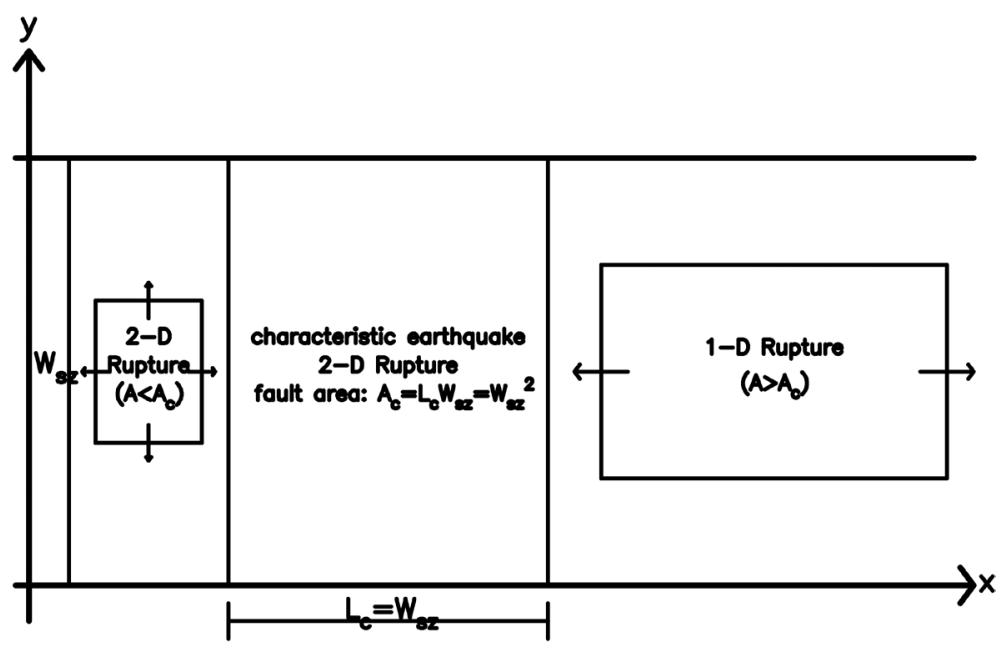

Fig. 2. Seismogenic zone with a thickness of $W_{s z}$ and the small, characteristic, and large earthquakes earthquake. The fault area of the characteristic earthquake has a fault length of $L_{c}\left(=W_{s z}\right)$ and a fault area of $A_{c}=L_{c} W_{s z}=W_{s z}{ }^{2}$. 
curve to intersect the point where $D_{o}$ and $L$ are both zero. The curves of the two functions result in a bigger reduction in uncertainties of the curve fit to the data points of SS events than a straight line.

For plate-boundary earthquakes, Leonard (2010) proposed four displacement models and obtained the following empirical relationships: (1) $D_{o} \sim L^{0.833}$ for DS events; and (2) $D_{o} \sim L^{1.0}$ when $A=0-3400 \mathrm{~m}^{2}, D_{o} \sim L^{0.833}$ when $A=3400$ $45000 \mathrm{~m}^{2}$, and $D_{o} \sim L^{0.5}$ when $A>45000 \mathrm{~m}^{2}$ for SS events. For earthquakes in stable continental regions, he obtained $D_{o} \sim L^{0.833}$ when $A>5500 \mathrm{~m}^{2}$ for all slip-type events. Based on the fault parameters inversed from finite-fault slip models for earthquakes with $M_{w}=5.4-9.2$, Thingbaijam et al. (2017) observed: (1) $D_{o} \sim L^{0.975 \pm 0.203}$ (with $S D=0.132$ ) for RE events; $D_{o} \sim L^{1.302} \pm 0.303$ (with $S D=0.252$ ) for NL events; $D_{o} \sim L^{0.879 \pm 0.144}$ (with $S D=0.276$ ) for SS events; and $D_{o} \sim L^{1.029 \pm 0.223}$ (with $S D=0.213$ ) for subduction- interface events; and (2) $D_{o} \sim W^{0.767 \pm 0.397}$ (with $S D=0.200$ ) for RE events; $D_{o} \sim W^{2.512} \pm 0.842$ (with $S D=0.223$ ) for NL events; $D_{o} \sim W^{2.391} \pm 0.485$ (with $S D=0.213$ ) for SS events; and $D_{o} \sim W^{1.244} \pm 0.577$ (with $S D=0.178$ ) for subduction-interface events. Considering a regional scale, they assumed that since the seismogenic depth is almost constant, the scaling behavior is corresponding to the $W$ model, thus meaning that $D_{o}$ is not correlated with $L$. They also observed that except for shallow crustal RE events, the ratio $D_{o} / W$ (the average strain drop) increases with $M_{w}$, suggesting scale- dependent strain drop and stress drop.

Stirling et al. (2002) obtained low dependence of $D_{o}$ on $L: D_{o} \sim L^{0.60 \pm 0.08}$ (with $S D=0.32$ ) for 95 instrumental events (post-1900); $D_{o} \sim L^{0.35 \pm 0.10}$ (with $S D=0.33$ ) for 30 pre-instrumental events (pre-1900). The two scaling exponents are smaller than that $(=0.88)$ estimated by Wells and Coppersmith (1994) as mentioned above. Clearly, there is low dependence of $D_{o}$ on $L$ from Stirling et al. (2002). Moreover, from the data of 50 events with $M_{w}=6.1-8.1$ censored from those of Wells and Coppersmith (1994), Stirling et al. (2002) obtained $D_{o} \sim L^{0.18 \pm 0.08}$ (with $S D=0.24$ ). This exhibits weak dependence of $D_{o}$ on $L$ an opposite to all the previous studies with strong dependence of $D_{o}$ on $L$.

\subsection{Modeling}

Shaw and Scholz (2001) proposed a model to interpret the observations as mentioned above. Their theoretical $D_{o}-L$ law is described below: $D_{o}=1 /(2 / L)$ when $L \leq 2 W$ and $D_{o}$ $=1 /[(1 / L)+1 / 2 W)]$ when $L>2 W$. Their observation and modeling are inconsistent with constant $\Delta \sigma$.

In order to examine the $D_{o}-L$ scaling law, Liu-Zeng et al. (2005) considered a simplified 1-D model of spatially heterogeneous slip, $D(x, y)$, which is characterized by a stochastic function with a Fourier spectrum that decays as $k^{-a}$, where $k$ is the wavenumber and $\alpha$ is a parameter representing the spatial smoothness of slip. They also assumed that an individual earthquake ruptures only along one spatially continuous segment. From their model, the scaling relation $D_{o} \sim L^{n}$ is related to the spatial heterogeneity of slip. The scaling exponent $\mathrm{n}$ increases with $\alpha$ in a form of $n \approx \alpha-0.5$ for $0.5<\alpha<1.5$, and the case with $n=1$ only occurs when $\alpha \approx$ 1.5 , which exhibits a relatively smooth spatial distribution of slip. Their simulation results show that $D_{o} / L$ is higher for the faults with large slip heterogeneity than those with spatially smooth slip. From the simulations based on spontaneous ruptures on multi-segmented SS faults in a 3-D half-space, Kase (2010) favored the empirical scaling law: a strong increase of $D_{o}$ with $L$ and $D_{o}$ tends to saturate for very long $L$.

The previous observational and theoretical studies all show that $D_{o}$ and $D_{\max }$ nonlinearly increase with $L$ in the power-law function and the increasing rates of $D_{o}$ and $D_{\max }$ with $L$ decrease with increasing with $L$. Together with observations, modeling results again implicate failure of the assumption of constant $\Delta \sigma$.

\section{7. $M_{o}-D_{o}$ SCALING RELATIONSHIPS}

Wells and Coppersmith (1994) observed $M_{o} \sim D_{o}^{1.34}$ (from $M_{w} \sim D_{o}^{0.89 \pm 0.09}$ with $S D=0.38$ ) and $M_{o} \sim D_{\max }^{1.17}$ (from $M_{w} \sim D_{\max }{ }^{0.78} \pm 0.06$ with $\left.S D=0.29\right)$ for SS events; $M_{o} \sim D_{o}^{0.20}$ (from $M_{w} \sim D_{o}^{0.13 \pm 0.36}$ with $S D=0.50$ ) and $M_{o} \sim D_{\max }^{0.66}$ (from $M_{w} \sim D_{\max }{ }^{0.44 \pm 0.26}$ with $S D=0.52$ ) for RE events; $M_{o} \sim D_{o}^{0.98}$ (from $M_{w} \sim D_{o}^{0.65 \pm 0.25}$ with $S D=0.33$ ) and $M_{o} \sim D_{\max }{ }^{1.07}$ (from $M_{w} \sim D_{\max } 0.71 \pm 0.15$ with $S D=0.34$ ) for NL events; $M_{o} \sim D_{o}^{1.23}$ (from $M_{w} \sim D_{o}^{0.65 \pm 0.25}$ with $S D=0.39$ ) and $M_{o} \sim D_{\max }{ }^{1.11}$ (from $M_{w} \sim D_{\max }{ }^{0.74 \pm 0.09}$ with $S D=0.40$ ) for all slip-type events from their large data base. For the source data from the finitefault rupture models of 31 world-wide earthquakes with $M_{o}$ $=2.2 \times 10^{17}-1.2 \times 10^{21} \mathrm{NM}$, Mai and Beroza (2000) obtained $M_{o} \sim D_{o}^{1.82}$ (from $D_{o} \sim M_{o}^{0.55 \pm 0.10}$ with $S D=0.20$ ) for 8 SS events; $M_{o} \sim D_{o}^{3.45}$ (from $D_{o} \sim M_{o}^{0.29 \pm 0.07}$ with $S D=0.26$ ) for $11 \mathrm{DS}$ events; and $M_{o} \sim D_{o}^{2.86}$ (from $D_{o} \sim M_{o}^{0.35 \pm 0.06}$ with $S D=0.24$ ) for 31 all slip-type events. Based on a global catalogue, Papazachos et al. (2004) obtained the following scaling relationships: (1) for SS events: $M_{o} \sim D_{o}^{2.21}$ for $M_{w}=6.0-8.0 ;$ (2) for DS events in continents: $M_{o} \sim D_{o}^{2.08}$ for $M_{w}=6.0-7.5,6.0 \leq M_{w} \leq 7.5$; and (3) for DS events in subduction regions: $M_{o} \sim D_{o}^{2.34}$ for $M_{w}=6.7-9.2$. From the source parameters inverted from finite-fault slip models of 19 events with $M_{w}=4.6-8.9$ (12 DS and 7 SS events) in Taiwan, Yen and Ma (2011) obtained $D_{o e}=1.68 \pm 0.33$ when $M_{o} \leq 10^{27}$ dyne-cm and $M_{o} \sim D_{o e}{ }^{3}$ for all events (from $D_{o e} \sim M_{o}^{0.08 \pm 0.14}$ with $S D=2.47$ for SS events; $D_{o e} \sim M_{o}^{0.20 \pm 0.13}$ with $S D=2.32$ for DS events; and $D_{o e} \sim M_{o}^{0.13 \pm 0.09}$ with $S D$ $=1.64$ for all events) when $M_{o}>10^{27}$ dyne-cm based on the effective average displacement, $D_{o e}$. $D_{o}$ is almost constant for the events with $M_{o} \leq 10^{27}$ dyne-cm. For 18 events with $M_{w}=5.9-8.2$ in New Zealand, Dowrick and Rhoades (2004) obtained $M_{o} \sim D_{o}{ }^{3}$. For global subduction-zone earthquakes with $M_{w}=6.7-9.2$, including 25 slip models of 10 
great earthquakes around Japan, Murotani et al. (2013) obtained $M_{o} \sim D_{o}{ }^{3}$ (from $D_{o} \sim M_{o}{ }^{1 / 3} \pm 1.64$ with $S D=1.72$ ). For the events with $M_{w}=5.4-9.2$, Thingbaijam et al. (2017) observed $M_{o} \sim D_{o}^{3.33}\left[\right.$ from $\log \left(D_{o}\right) \sim(0.451 \pm 0.093) M_{w}$ with $S D$ $=0.149$ ] for RE events; $M_{o} \sim D_{o}^{2.16}$ [from $\log \left(D_{o}\right) \sim(0.693 \pm$ $0.066) M_{w}$ with $S D=0.195$ ] for NL events; $M_{o} \sim D_{o}^{2.67}$ [from $\log \left(D_{o}\right) \sim(0.558 \pm 0.054) M_{w}$ with $\left.S D=0.227\right]$ for SS events; and $D_{o} \sim M_{o}^{2.72}$ [from $\log \left(D_{o}\right) \sim(0.552 \pm 0.067) M_{w}$ with $S D=$ $0.171]$ for the events on the subduction interface.

The previous studies suggest that for all slip-type faults $M_{o}$ scales with $D_{o}$ almost in a form of $M_{o} \sim D_{o}{ }^{n}$ with $2 \leq n \leq 3$ and the scaling exponent $n$ is smaller for DS events than for $\mathrm{SS}$ events. The results of this section seem able to be consistent with the definition of $M_{o}=m D_{o} L W$ by Aki (1966).

\section{8. $D_{o}$ - $A$ SCALING RELATIONSHIPS}

Leonard (2010) obtained $D_{o} \sim A^{0.5}$ for all slip-type earthquakes in plate boundary. For the source data from the finite-fault rupture models of earthquakes with $M_{w}=5.4-9.2$, Thingbaijam et al. (2017) observed $D_{o} \sim A^{0.429 \pm 0.134}$ (with $S D$ $=0.180$ ) for RE events; $D_{o} \sim A^{0.858 \pm 0.214}$ (with $S D=0.330$ ) for NL events; $D_{o} \sim A^{0.597 \pm 0.112}$ (with $S D=0.302$ ) for SS events; and $D_{o} \sim A^{0.582 \pm 0.136}$ (with $S D=0.257$ ) for subduction-interface events. The studies of the two groups essentially suggest $D_{o} \sim A^{0.5}$, thus indicating $D_{o} \sim L$ or $D_{o} \sim W$, for all slip-type events. Moreover, Thingbaijam et al. (2017) stressed that the NL events somewhat deviate from the scaling law.

\section{9. $M_{o^{-}} A$ SCALING RELATIONSHIPS}

In principle, Eq. (2) leads to $M_{o} \sim A^{1.5}$ for constant $\Delta \sigma$. From world-wide events with $M_{s}=5.8-8.5$, Wyss (1979) obtained $M_{o} \sim A^{1.5}$. From 88 worldwide continental SS events with $M_{w}=5-8$, Hanks and Bakun $(2002,2008)$ obtained $M_{o} \sim A^{1.5}\left[\right.$ from $\left.M_{w}=\log (A)+3.98 \pm 0.03\right]$ for $A<537 \mathrm{~km}^{2}$; and $M_{o} \sim A^{2}\left[\right.$ from $\left.M_{w}=\log (A)+3.07 \pm 0.04\right]$ for $A>537 \mathrm{~km}^{2}$, thus suggesting a change of the scaling relationships at $A<$ $537 \mathrm{~km}^{2}$. This meets the effect as displayed in Fig. 2. From 26 world-wide events with all slip-type faulting with $M_{w}$ $=5.4-8.0$, Ichinose et al. (2006) obtained $M_{o} \sim A_{a}{ }^{1.75}$ (from $\left.A_{a} \sim M_{o}^{0.57 \pm 0.06}\right)$, where $A_{a}$ is the combined area of asperities in $\mathrm{km}^{2}$. For the source data obtained the finite-fault rupture models of world-wide earthquakes, Irikura and Miyake (2011) found $M_{o} \sim A_{e}^{1.5}$ (from $A_{e} \sim M_{o}^{2 / 3}$ ) for $M_{o}<7.5 \times 10^{18}$ $\mathrm{N}-\mathrm{m}$ and $M_{o} \sim \mathrm{A}^{2}$ (from $A_{e} \sim M_{o}^{1 / 2}$ ) for $M_{o}>7.5 \times 10^{18} \mathrm{~N}-\mathrm{m}$. For 32 slip models of world-wide subduction-zone earthquakes with $M_{w}=6.7-9.2$, including 25 slip models of 10 great earthquakes around Japan, Murotani et al. (2013) obtained $M_{o} \sim A^{1.5}$ (from $A \sim M_{o}^{2 / 3}$ with $S D=1.61$ ). They also obtained $M_{o} \sim A_{a}^{1.5}$ (from $A_{a} \sim M_{o}^{2 / 3}$ with $S D=1.78$ ) and $A_{a} / A$ $=0.2$ where $A_{a}$ to be the area of sub-faults having displacements greater than 1.5 times $D_{o}$. From a large number of all slip-type earthquakes with $M_{w}=5.4-9.2$, Thingbai- jam et al. (2017) obtained $M_{o} \sim A^{1.43}$ [from $\log (A) \sim(1.049 \pm$ $0.066) M_{w}$ with $S D=0.121$ ] for RE events; $M_{o} \sim A^{1.86}$ [from $\log (A) \sim(0.808 \pm 0.059) M_{w}$ with $\left.S D=0.181\right]$ for NL events; $M_{o} \sim A^{1.59}\left[\right.$ from $\log (A) \sim(0.942 \pm 0.058) M_{w}$ with $\left.S D=0.184\right]$ for SS events; and $M_{o} \sim A^{1.58}[$ from $\log (A) \sim(0.949 \pm 0.049)$ $M_{w}$ with $\left.S D=0.150\right]$ for subduction-interface events. The results suggest self-similar $\log \left(M_{o}\right)-\log (A)$ scaling relationship for all the cases, except for NL events.

There are some studies of $M_{o}-A$ scaling relationships from regional data. For the source data obtained the finitefault rupture models of 19 Taiwan's earthquakes with $M_{w}=$ 4.6 - 8.9, $A_{e}$, Yen and Ma (2011) obtained $M_{o} \sim A_{e}{ }^{1.25}$ (from $A_{e} \sim M_{o}^{0.80 \pm 0.13}$ with $S D=0.43$ ), where $A_{e}$ is the effective ruptured area of asperities in $\mathrm{km}^{2}$, for $12 \mathrm{DS}$ events; $M_{o} \sim A_{e}{ }^{1.09}$ (from $A_{e} \sim M_{o}^{0.92 \pm 0.14}$ with $S D=0.40$ ) for $8 \mathrm{SS}$ events; and $M_{o} \sim A_{e}^{1.15}$ (from $A_{e} \sim M_{o}^{0.87 \pm 0.09}$ with $S D=0.41$ ) for 19 all sliptype events. Clearly, the value of scaling exponent estimated by Yen and Ma (2011) is lower than the expected one (= $1.5)$ and those obtained by others. This might suggest particular source properties of Taiwan's earthquakes. It would significant to study this problem from a larger data set.

In addition, the $M_{o}-A$ scaling relationships based on Eq. (4) can be transferred from the $M_{w}-A$ relationships inferred by some authors. Wells and Coppersmith (1994) obtained $M_{o} \sim A^{1.53}$ [from $M_{w} \sim(1.02 \pm 0.03) \log (A)$ with $S D=0.23$ ] for 83 SS events; $M_{o} \sim A^{1.35}$ [from $M_{w} \sim(0.90 \pm 0.05) \log (A)$ with $S D$ $=0.25]$ for $43 \mathrm{RE}$ events; $M_{o} \sim A^{1.53}$ [from $M_{w} \sim(1.02 \pm 0.10)$ $\log (A)$ with $S D=0.25$ ] for $22 \mathrm{NL}$ events; and $M_{o} \sim A^{1.47}$ [from $M_{w} \sim(0.98 \pm 0.03) \log (A)$ with $\left.S D=0.24\right]$ for 148 all sliptype events. For the source data from the finite-fault rupture models of 31 world-wide earthquakes with $M_{o}=2.2 \times 10^{17}$ - $1.2 \times 10^{21} \mathrm{Nm}$, Mai and Beroza (2000) obtained $M_{o} \sim A^{1.75}$ (from $A \sim M_{o}^{0.44 \pm 0.11}$ with $S D=0.21$ ) for $8 \mathrm{SS}$ events; $M_{o} \sim A^{1.33}$ (from $A \sim M_{o}^{0.71 \pm 0.07}$ with $S D=0.26$ ) for $11 \mathrm{DS}$ events; and $M_{o} \sim A^{1.39}$ (from $A \sim M_{o}^{0.64 \pm 0.06}$ with $S D=0.25$ ) for 31 all sliptype events. From 15 inland world-wide crustal events, with majority in California, with $M_{w}=5.7-7.2$ for all slip-type events, Somerville et al. (1999) obtained $M_{o} \sim A^{1.5}$. From 7 NL events with $M_{w}=5.9-7.1$ in New Zealand, Villamor et al. (2001) obtained $M_{o} \sim A^{1.99}$. Based on 396 world-wide events with $M_{w}=6.1-8.1$ by Wells and Coppersmith (1994), Stirling et al. (2002) obtained $M_{o} \sim A^{1.685}$ [from $M_{w} \sim(0.89 \pm$ $0.05) \log (A)$ with $S D=0.31]$ from 108 instrumental events and $M_{o} \sim A^{1.923}$ [from $M_{w} \sim(0.78 \pm 0.06) \log (A)$ with $S D=0.16$ ] from 30 pre-instrumental events. From 50 censored data, they obtained $M_{o} \sim A^{2.055}$ [from $M_{w} \sim(0.73 \pm 0.7) \log (A)$ with $S D=0.26]$. Based on 396 world-wide events with $M_{w}=6.1$ - 8.1 by Wells and Coppersmith (1994), Stirling et al. (2002) obtained $M_{o} \sim A^{1.335}$ [from $\left.M_{w} \sim(0.89 \pm 0.05) \log (A)\right]$ from 108 instrumental events and $M_{o} \sim A^{1.17}$ [from $M_{w} \sim(0.78 \pm 0.06)$ $\log (A)]$ from 30 pre-instrumental events. From 47 censored data, they obtained $M_{o} \sim A^{1.095}\left[\right.$ from $\left.M_{w} \sim(0.73 \pm 0.07) \log (A)\right]$. From 15 events with $M_{\mathrm{w}}=5.9-8.2$ in New Zealand, Dowrick and Rhoades (2004) obtained $M_{o} \sim A^{1.5}$ [from $\log (A) \sim 1.0 M_{w}$ ]. 
Based on world-wide events, Papazachos et al. (2004) obtained $M_{o} \sim A^{1.83}$ for SS events with $M_{w}=6.0-8.0 ; M_{o} \sim A^{1.92}$ or DS events with $M_{w}=6.0-7.5$ in continents; and $M_{o} \sim A^{1.74}$ for DS events with $M_{w}=6.7-9.2$ in subduction regions. From 16 worldwide large SS events with $M_{w}=5.7-7.9$, Somerville et al. (2006) obtained $M_{o} \sim A^{1.58}$. Leonard (2010) obtained $M_{o} \sim A^{1.5}$ for SS events, $M_{o} \sim A^{1.5}$ for DS events, and $M_{o} \sim A^{1.5}$ for events in stable continental regions. Strasser et al. (2010) obtained $M_{o} \sim A^{1.30}$ [from $M_{w} \sim(0.846 \pm 0.046)$ $\log (A)$ with $S D=0.277]$ for 85 interface events with $M_{w}=$ $6.3-9.4$ and $M_{o} \sim A^{1.47}$ [from $M_{w} \sim(0.981 \pm 0.093) \log (A)$ with $S D=0.813$ ] for 18 intraslab events with $M_{w}=6.3$ - 9.4.

The values of scaling exponent $n$ of the relationship of $M_{o} \sim A^{n}$ obtained by various authors are listed in Table 1 . All observations show $n \approx 1.5$ and thus confirm the theoretically expected $M_{o}-A$ scaling relationship, i.e., $M_{o} \sim A^{1.5}$. Meanwhile, except for the results by Hanks and Bakun $(2002,2008)$, the scaling relationship holds in a large range of $M_{o}$ or $M_{w}$.

For SS earthquakes in Japan, there are two end-member regimes in the $M_{o}-A$ scaling relationships: $M_{o} \sim A^{1.5}$ for small (self-similar) earthquakes and $M_{o} \sim A$ for very large $(W$ model) events. The transition between these two regimes, there is a transition regime with $M_{o} \sim A^{n}$ where $n$ is between 1 and $3 / 2$. But, some seismologists proposed $n>3 / 2$ (see Luo et al. 2017; and cited references therein). For an example, Irikura and Miyake (2011) assumed $n=2$ for intermediate magnitudes mentioned above. Based on earthquake cycle simulations, analytical dislocation models and numerical crack models on SS faults, Luo et al. (2017) investigate the mechanism of this transition regime. They found that, even if under the assumption of constant $\Delta \sigma$, the major factors in controlling properties of the transition regime are the surface rupture effects, comprising an effective rupture elongation along-dip due to a mirror effect and systematic changes of the shape factor relating slip to stress drop. In addition, the secondary factors are deep rupture penetration, deeper viscous layer and scale-dependency of stress drop. Based on physical modeling, they proposed a simplified formula to describe the effects in $M_{o}$ - $A$ scaling relations for SS earthquakes in a large range of magnitudes.

\section{0. $L$ - $W$ RELATIONSHIPS}

Based on the self-similarity proposed by Kanamori and Anderson (1975), $W$ is proportional to $L$. Wang and Ou (1998) observed (1) for the surface rupture length: $W \sim L^{0.38 \pm 0.23}$ when $L<20 \mathrm{~km}$ and $W \sim L^{0.05 \pm 0.10}$ when $L>20$ $\mathrm{km}$ for all slip-type events; and (2) for the subsurface rupture length: $W \sim L^{0.99} \pm 0.37$ when $L<20 \mathrm{~km}$ and $W \sim L^{0.09 \pm 0.09}$ when $L>20 \mathrm{~km}$ for all slip-type events. Obviously, $W$ is independent upon $L$ when $L>20 \mathrm{~km}$, which is almost the average thickness of seismogenic zone in Taiwan. Leonard (2010) obtained $W \sim L^{0.67}$ for DS events, SS events with $A=$
$3400-45000 \mathrm{~m}^{2}$ in plate boundary, and all slip-type events with $A>2500 \mathrm{~m}^{2}$ in stable continental regions. For Taiwan's earthquakes, Yen and Ma (2011) observed a linear increase in $W$ with $L$. In addition, Wesnousky (2008) observed that the aspect ratio $L / W$ is well correlated to $L: L / W \sim L^{b}$, with $b=$ 0.96 for SS events, $b=0.81$ for NL events, and $b=0.55$ for RE events. Thingbaijam et al. (2017) observed that $L$ grows more rapidly with $M_{o}$ than $W ; L / W$ increase with $M_{o}$, with an increasing rate increasing from RE events, to NL events, to SS events. They also found that subduction-interface earthquakes have wider $W$ (thus resulting a larger rupture area $A$ ) than others. Irikura and Miyake (2011) observed that $W=L$ when $W<W_{s z}$ and $W=W_{s z} / \sin (\theta)$ when $W \geq W_{s z}$ where $W_{s z}$ and $\theta$ are the width of seismogenic-zone and the dip angle of a fault, respectively.

\section{1. $M_{o}-L$ AND $M_{o}-W$ RELATIONSHIPS}

\subsection{Observations of the $M_{o}-L$ Relationship}

Based on the definition of $M_{o}$, the $M_{o}-L$ and $M_{o}-W$ scaling relationships are naturally important for exploring scaling of faults. First, the $M_{o}-L$ scaling relationship is taken into account. It is interesting to ask a question if the scaling relationship holds for the whole range of $M_{o}$ as well as $M_{w}$ or not. Hanks (1977) considered that $M_{o}-L$ scaling holds for a wide range of events with $L$ varying from meters to hundreds of kilometers. Actually, there is a large scatter in the data points of $M_{o}$ versus $L$ between $\Delta \sigma=1$ bar to $\Delta \sigma=100$ bars, and there are systematic deviations for very small events. Hank's plots of $M_{o}$ versus $L$ show the scaling exponent, $n$, is $n>3$ for $L<0.5 \mathrm{~km}$ and $n \approx 3$ for $L>$ $0.5 \mathrm{~km}$. Hence, $M_{o}$ is almost independent upon $L$ for $L<$ $0.5 \mathrm{~km}$. From this observation, Aki (1987) proposed that there is a lower-bound magnitude of $M_{l}=3$ for earthquakes. But, this is questionable. Based on Aki's proposition, the values of $M_{o}$ for smaller earthquakes are lower than those estimated from the $M_{o}-L$ relation, which is inferred from larger events. This could be due to under-estimates of $M_{o}$ for smaller events because of weak long-period signals. Gutenberg and Richter (1956) reported $\log \left(E_{s}\right)=11.8+1.5 M_{w}$ ( $E_{s}$ in ergs) or $\log \left(E_{s}\right)=4.8+1.5 M_{w}$ (in J). If $M_{l}$ exists, $E_{s}$ will keeps constant and does not decrease with $M_{w}$ when $M_{w}<M_{l}$. However, Wang (2015) observed that $E_{s}$ decreases with $M_{w}$ down to $M_{w}=0$. This means that the lower limit of earthquake size does not exist. Scholz et al. (1986) stated that large intraplate earthquakes consistently have greater $M_{o} / L$ than interplate events, the difference being about a factor of five. Aki (1992) observed a deviation of the $M_{o}-L$ correlation for California earthquakes from the average one for Japanese events. However, the deviation is larger for SS events and smaller for RE events.

Nuttli (1983a) obtained $M_{o} \sim L^{3.65}$ in a large range of $M_{o}$. From 10 worldwide events with $M_{w}=5.46-7.79$ and $h=$ 3 - $140 \mathrm{~km}$, Johnston et al. (1994) obtained $M_{o} \sim L^{2.04}$. From 





65 NL events with $M_{w}=6.5-7.2$, Mason (1996) obtained $M_{o} \sim L^{1.98}$. Wells and Coppersmith (1994) observed $M_{o} \sim L_{o}^{1.68}$ [from $M_{w} \sim(1.12 \pm 0.08) \log \left(L_{o}\right)$ with $S D=0.28$ and $M_{o} \sim L_{s}^{1.11}$ [from $M_{w} \sim(1.49 \pm 0.05) \log \left(L_{s}\right)$ with $S D=0.24$ ] $\left(L_{o}=\right.$ surface rupture length and $L_{s}=$ subsurface rupture length) for SS events, $M_{o} \sim L_{o}{ }^{1.83}$ [from $M_{w} \sim(1.22 \pm 0.16) \log \left(L_{o}\right)$ with $S D$ $=0.28$ ] and $M_{o} \sim L_{s}^{0.95}$ [from $M_{w} \sim(1.49 \pm 0.05) \log \left(L_{s}\right)$ with $S D=0.24]$ for RE events, $M_{o} \sim L_{o}^{1.98}$ [from $M_{w} \sim(1.32 \pm 0.26)$ $\log \left(L_{o}\right)$ with $S D=0.34$ ] and $M_{o} \sim L_{s}^{0.75}$ [from $M_{w} \sim(1.54 \pm$ 1.18) $\log \left(L_{s}\right)$ with $S D=0.31$ ] for NL events, $M_{o} \sim L_{o}^{1.74}$ [from $M_{w} \sim(1.16 \pm 0.07) \log \left(L_{o}\right)$ with $\left.S D=0.28\right]$ and $M_{o} \sim L_{s}^{1.04}$ [from $M_{w} \sim(1.54 \pm 0.18) \log \left(L_{s}\right)$ with $\left.S D=0.26\right]$ for all slip-type events with $M_{w}=5.8-8.9$. From 200 world-wide events with $M_{w}=4.3-8.5$, Wang and $\mathrm{Ou}(1998)$ found $M_{o} \sim L^{1.98 \pm 0.14}$ $\approx L^{2}$ in a large range of $M_{o}$. For DS earthquakes, Stock and Smith (2000) obtained $M_{o} \sim L^{3.2}$ in Japan and $M_{o} \sim L^{2.9}$ in eastern Russia. For the source data from the finite-fault rupture models of 31 world-wide earthquakes with $M_{o}=2.2 \times 10^{17}$ $-1.2 \times 10^{21} \mathrm{NM}$, Mai and Beroza (2000) obtained $M_{o} \sim L^{2.78}$ (from $L \sim M_{o}^{0.36 \pm 0.06}$ with $S D=0.11$ ) for 8 SS events; $M_{o} \sim L^{2.63}$ (from $L \sim M_{o}^{0.38 \pm 0.05}$ with $S D=0.18$ ) for $11 \mathrm{DS}$ events; and $M_{o} \sim L^{2.86}$ (from $L \sim M_{o}^{0.35 \pm 0.04}$ with $S D=0.16$ ) for 31 all sliptype events.

For 396 world-wide events with $M_{w}=6.1-8.1$, Stirling et al. (2002) obtained $M_{o} \sim L^{1.2}$ [from $M_{w} \sim(0.95 \pm 0.06)$ $\left.\log \left(L_{o}\right)\right]$. For SS events with $M_{w} \geq 5.5$, Romanowicz and Ruff (2002) obtained $M_{o} / M_{\text {omin }} \sim L^{n}$, where $M_{\text {omin }}=0.5 \times 10^{27}$ dyne-cm and $n=1.2 \pm 1.4$ for continental/interplate events and $M_{\text {omin }}=1.0 \times 10^{27}$ dyne-cm and $n=1.09 \pm 2.4$ for continental/intraplate events. Papazachos et al. (2004) obtained $M_{o} \sim L^{2.54}$ for SS events with $M_{w}=6.0-8.0 ; M_{o} \sim L^{3.00}$ for DS events with $M_{w}=6.0-7.5$ in continents; and $M_{o} \sim L^{2.73}$ for DS events with $M_{w}=6.7-9.2$ in the subduction regions.

For world-wide earthquakes with $M_{o}>10^{25}$ dyne-cm and $L>15 \mathrm{~km}$, Wesnousky (2008) obtained $M_{o} \sim L^{1.31}$ for SS events, $M_{o} \sim L^{0.71}$ for NL events, $M_{o} \sim L^{2.82}$ for RE events, and $M_{o} \sim L^{1.52}$ for all slip-type faults. It is noted that the data points for two small events with $M_{w}<10^{25.3}$ dyne-cm or $M_{w}<6.2$ remarkably depart from his linear relationship of $\log \left(M_{o}\right)$ versus $\log (L)$. Obviously, the scaling exponents vary with slip types and are all smaller than 2 . He also observed $L / W \sim L^{b}: b=0.96$ for SS events, $b=0.81$ for NL events, and $b=0.55$ for RE events.

Strasser et al. (2010) obtained $M_{o} \sim L^{2.09}$ [from $M_{w}=$ $(1.392 \pm 0.064) \log (L)$ with $S D=0.814]$ for interface events with $M_{w}=6.3-9.4$ and $M_{o} \sim L^{2.17}$ [from $M_{w}=(1.445 \pm 0.164)$ $\log (L)$ with $S D=0.813]$ for intraslab events with $M_{w}=6.3$ - 9.4. From world-wide events, Blaser et al. (2010) obtained $M_{o} \sim L^{2.42}$ for RE events with $M_{w}=6.1-9.5$ and $L=13-1400$ $\mathrm{km} ; M_{o} \sim L^{2.42}$ for SS events with $M_{w}=5.3-8.1$ and $L=7$ - $350 \mathrm{~km}$; and $M_{o} \sim L^{2.78}$ for all slip-type events with $M_{w}=$ 5.3 - 9.5 and $L=7-1400 \mathrm{~km}$. From world-wide events $\left(M_{w}\right.$ $=4.7-9.0$ ) of all slip types, Funning et al. (2013) obtained $M_{o} \sim L^{2.0}$ for RE events, $M_{o} \sim L^{1.6}$ for SS events, and $M_{o} \sim L^{1.8}$ for all slip-type events. Based on 396 world-wide events with $M_{w}=6.1-8.1$ by Wells and Coppersmith (1994), Stirling et al. (2002) obtained $M_{o} \sim L^{1.425}$ [from $M_{w} \sim(0.95 \pm 0.06) \log (L)$ with $S D=0.37$ f from 167 instrumental events and $M_{o} \sim L^{1.125}$ [from $M_{w} \sim(0.75 \pm 0.06) \log (L)$ with $S D=0.21$ ] from 59 preinstrumental events. From 50 censored data, they obtained $M_{o} \sim L^{1.3}$ [from $M_{w} \sim(0.80 \pm 0.10) \log (L)$ with $S D=0.30$ ].

From microearthquakes with $M_{o}=10^{16}-10^{21}$ dyne-cm recorded at Mt. Etna volcano and those with $M_{o}=10^{17}-10^{20}$ dyne-cm in the Calabrian Arc, Southern Italy, Patanè et al. (1997) observed a linear relationship between $\log \left(M_{o}\right)$ and $\log (R)$, where $R$ is the radius of an event and also a type of fault length, $L$, in the whole range of RE events though they did not show the scaling exponent.

On the other hand, some authors observed a change of the $M_{o}-L$ scaling relationship changes from small to large events. For Japanese events, Shimazaki (1986) found a change from $M_{o} \sim L^{3}$ to $M_{o} \sim L^{2}$ scaling at $M_{o}=7.5 \times 10^{25}$ dyne$\mathrm{cm}$ or $M_{w}=6.5$. The separation point is related to $L=17 \mathrm{~km}$, which is nearly the average thickness of seismogenic layer in Japan. The characteristic area $A_{c}$ is $17 \times 17 \mathrm{~km}^{2}$. This implicates that small Japanese events (with $M_{w}<6.5$ or $A<A_{c}$ ) are not similar to large ones (with $M_{w}>6.5$ or $A>A_{c}$ ). For world-wide SS events, Stirling et al. (1996) obtained $M_{o} \sim L^{5.0}$ when $L<50 \mathrm{~km}$ and $M_{o} \sim L^{1.3}$ when $L>50 \mathrm{~km}$. They also obtained $M_{o} \sim L^{2.1}$ for large intraplate earthquakes in Japan.

For 18 events with $M_{w}=5.9-8.2$ in New Zealand, Dowrick and Rhoades (2004) obtained $M_{0} \sim L^{3}$ [from $\log (L) \sim 0.5 M_{w}$ ] when $L<6.0 \mathrm{~km}$ and $M_{o} \sim L^{2.30}$ [from $\log (L) \sim(0.63 \pm 0.04) M_{w}$ ] when $L \geq 6.0 \mathrm{~km}$ where $L$ is the subsurface rupture length. Obviously, the value of $L=6 \mathrm{~km}$ to separate small and large event in New Zealand is much shorter than that in Japan. From a large number of different slip-type earthquakes with $M_{w}=5.4-9.2$, Thingbaijam et al. (2017) obtained $M_{o} \sim L^{2.44}[$ from $\log (L) \sim(0.614 \pm$ $0.043) M_{w}$ with $S D=0.083$ ] for RE events, $M_{o} \sim L^{3.09}$ [from $\log (L) \sim(0.485 \pm 0.036) M_{w}$ with $\left.S D=0.128\right]$ for NL events, $M_{o} \sim L^{2.20}\left[\right.$ from $\log (L) \sim(0.681 \pm 0.052) M_{w}$ with $\left.S D=0.151\right]$ for SS events, and $M_{o} \sim L^{2.57}\left[\right.$ from $\log (L) \sim(0.583 \pm 0.037) M_{w}$ with $S D=0.107]$ for subduction-interface events. They also gained $M_{o} \sim L^{3.13}$ for $M_{w} \leq 7.1$ and $M_{o} \sim L^{1.76}$ for $M_{w}>7.1$.

Romanowicz (1992) reported two different scaling laws for small and large strike-slip events, separating at $M_{o}$ $=10^{27}$ dyne-cm: $M_{o} \sim L^{1 / 2}$ for small events and $M_{o} \sim L$ for large ones. From a large data set of world-wide earthquakes with $M_{w}=4.0-9.6, M_{o}=10^{22}-10^{31}$ dyne-cm, $L=0.1-1000 \mathrm{~km}$, and $W=1-300 \mathrm{~km}$, Stock and Smith (2000) obtained: (1) for NL events: $M_{o} \sim L^{3.1}$ (with $S D=0.8$ ) for 32 small events and $M_{o} \sim L^{4.1}$ (with $S D=2.1$ ) for 6 large events; (2) for RE events: $M_{o} \sim L^{2.7}$ (with $S D=0.9$ ) for 77 small events and $M_{o} \sim L^{2.9}$ (with $S D=0.9$ ) for 9 large events; (3) for SS events in California: $M_{o} \sim L^{2.8}$ (with $S D=1.4$ ) for 27 small events and $M_{o} \sim L^{2.1}$ (with $S D=1.4$ ) for 9 large events; and (4) for SS events in other regions: $M_{o} \sim L^{2.9}$ (with $S D=1.1$ ) for 33 small 
events and $M_{o} \sim L^{2.3}$ (with $S D=1.4$ ) for 29 large events. But, for the earthquakes in Japan and eastern Russia, they obtained only a single linear relationship: (1) for 21 DS events in Japan: $M_{o} \sim L^{3.2}$ (with $S D=1.1$ ); and (2) for 16 DS events in eastern Russia: $M_{o} \sim L^{2.9}$ (with $S D=1.3$ ). Since the Japanese earthquakes used by them have $M_{o}<1.0 \times 10^{22} \mathrm{Nm}$, their data cover the range from small to large events used by Shimazaki (1986). Hence, their scaling law is essentially different from that inferred by Shimazaki (1986) in a wide $M_{o}$ range. Based on the subsurface rupture lengths of finitefault rupture models of 19 Taiwan's earthquakes with $M_{o}=$ 4.6 - 8.9, Yen and Ma (2011) obtained linear relationships: (1) for 12 DS events: $M_{o} \sim L^{2.38}$ (from $L \sim M_{o}^{0.42 \pm 0.06}$ with $S D$ $=0.19$ ); and (2) for 7 SS events: $M_{o} \sim L^{2.0}$ (from $L \sim M_{o}^{0.50 \pm 0.07}$ with $S D=0.20$ ); (3) for 19 all slip-type events: $M_{o} \sim L^{2.13}$ (from $L \sim M_{o}^{0.47 \pm 0.04}$ with $S D=0.19$ ). The reasons to yield linear relationships of $M_{o}$ versus $L$ in a large magnitude range for regional events in Japan and eastern Russia by Stock and Smith (2000) and for those in Taiwan by Yen and Ma (2011) are unclear, because the existence of a particular value of $M_{o}$ or $M_{w}$, which is associated with the seismogenic-zone depth in a region, to separate the two ranges of events is physically reasonable as mentioned below.

The values of $n$ of $M_{o} \sim L^{n}$ are listed in Table 2. The value of $n$ does not change from small to large world-wide events. On the other hand, the value of $n$ changes from small to large regional events of all slip types, and its value is larger for small events than for large ones for most of studies. Of course, there are opposite results for some studies as mentioned above [e.g., Stock and Smith (2000) for Japanese DS earthquakes and Yen and Ma (2011) for Taiwan's events]. According to the seismogenic-zone model, for regional earthquakes two scaling laws separated at a particular magnitude, which is related to the characteristic event having a rupture area of $A_{c}$, is more reasonable than a single scaling law. The presence of a single scaling law might be due to uncertainties of estimates of $L$. However, the problem is still open. Anyway, for each slip type the rupture length or width associated with the separating point seems to be consistent with the seismogenic-zone thickness. Considering the values of $n$ for all slip-type events, Table 2 cannot give us a substantial conclusion because the estimated values vary very much. This might be due to a small number of data in some studies. For the world-wide situation, earthquakes occur in different seismogenic zones with different depths and thus the single regression equation in a wide magnitude range represents a total effect of different seismogenic zones.

Anderson et al. (1996) addressed a significant problem whether the slip rate on a fault, $\gamma_{F}$, influences estimates of $M_{w}$ of shallow continental surface rupturing earthquakes. Based on 43 event from Wells and Coppersmith (1994), they suggested that the estimate of $M_{w}$ from $L$ can be improved by incorporating $\gamma_{F}$ with the linear equation under constant $\Delta \sigma: M_{w}=5.12+1.16 \log (L)-0.20 \log \left(\gamma_{F}\right)$. Nev- ertheless, from the formulae the influence on $M_{w}$ due to $\gamma_{F}$ is small, because the related coefficient $(=0.20)$ is smaller than that $(=1.16)$ for $\log (L)$. By using 80 events (including 56 SS, 13 RE, and 11 NL events), Anderson et al. (2017) found that when $\gamma_{F}$ is taken into account, the estimates of $M_{w}$ from $L$ are improved for SS events, yet not for the other two slip-type events. Their results are slightly different from those by Anderson et al. (1996). Based on a database of 80 events, including 56 SS, 13 RE, and 11 NL events, Anderson et al. (2017) re-evaluated the relationship of Model 1. Their results reveal that the value of $M_{w}$ calculated from $L$ are improved for SS earthquakes when slip rate is included but not for RE or NL events. Regardless of the $\gamma_{F}$ term, a linear model with $M_{w} \sim \log (L)$ over all rupture lengths implies that $\Delta \sigma$ depends on $L$. This is not supported by teleseismic observations. Hence, they proposed two more complicated models to correlate $M_{w}$ : Model 2 is a bilinear model with constant $\Delta \sigma$ for each linear section of the scaling relationship; and Model 3 is a bilinear model with constant $\Delta \sigma$ and derived from the model of Chinnery (1964). (The equations related to the two models can be found their paper and are not given here.) They prefer Model 3 because it is specified with constant $\Delta \sigma$ over the entire range of $L$ for any $\gamma_{F}$ and fits the data very well. The three models are all dependent on $\gamma_{F}$ for SS events. The dependence on $\gamma_{F}$ from observations supports the conclusion that for SS events of a given length, $\Delta \sigma$ decreases with increasing $\gamma_{F}$. Based on the estimates of magnitudes of the $2016 M_{w} 7.8$ Kaikoura and 2010 $M_{w} 7.1$ Darfield earthquakes in New Zealand, Stirling and Anderson (2018) supported the result obtained by Anderson et al. (2017).

\subsection{Observations of the $M_{o}-W$ Relationship}

Some $M_{o}-W$ scaling relationships are described below. Wells and Coppersmith (1994) obtained: (1) $M_{o} \sim W^{2.24}$ [from $M_{w} \sim(2.59 \pm 0.18) \log (W)$ with $S D=0.45$ ] for $87 \mathrm{SS}$ events; (2) $M_{o} \sim W^{2.24}$ [from $M_{w} \sim(1.95 \pm 0.15) \log (W)$ with $S D=0.32$ ] for $43 \mathrm{RE}$ events; (2) $M_{o} \sim W^{3.45}$ [from $M_{w} \sim(2.11$ $\pm 0.28) \log (W)$ with $S D=0.31]$ for $23 \mathrm{NL}$ events; and (4) $M_{o} \sim W^{2.24}\left[\right.$ from $M_{w} \sim(2.25 \pm 0.12) \log (W)$ with $\left.S D=0.41\right]$ for 153 all slip-type events. For the source data from the finitefault rupture models of 31 world-wide earthquakes with $M_{o}$ $=2.2 \times 10^{17}-1.2 \times 10^{21} \mathrm{NM}$, Mai and Beroza (2000) obtained $M_{o} \sim W^{11.11}$ (from $W \sim M_{o}^{0.09 \pm 0.06}$ with $S D=0.12$ ) for 8 SS events; $M_{o} \sim W^{3.03}$ (from $W \sim M_{o}^{0.33 \pm 0.03}$ with $S D=0.16$ ) for 11 DS events; and $M_{o} \sim W^{3.45}$ (from $W \sim M_{o}^{0.29 \pm 0.04}$ with $S D$ $=0.18)$ for 31 all slip-type events. For the events with $M_{w}$ $=5.9-8.2$ in New Zealand, Dowrick and Rhoades (2004) obtained $M_{o} \sim W^{3}$ when $L<6.0 \mathrm{~km}$ and $M_{o} \sim W^{4.29}$ when $L \geq$ 6.0. Papazachos et al. (2004) obtained: (1) $M_{o} \sim W^{6.52}$ for SS events with $M_{w}=6.0-8.0$; (2) $M_{o} \sim W^{5.36}$ for DS events with $M_{w}=6.0-7.5$ in continents; and (3) $M_{o} \sim W^{5.00}$ for DS event with $M_{w}=6.7-9.2$ in subduction regions. Strasser et al. 
(2010) obtained $M_{o} \sim W^{2.09}\left[\right.$ from $M_{w}=(1.805 \pm 0.151) \log (W)$ with $S D=0.392]$ for interface events with $M_{w}=6.3-9.4$ and $M_{o} \sim W^{2.17}$ [from $M_{w}=(2.511 \pm 0.217) \log (W)$ with $S D=$ $0.178]$ for intraslab events with $M_{w}=6.3-9.4$. Blaser et al. (2010) obtained $M_{o} \sim W^{3.33}$ for RE events with $M_{w}=6.1-9.5$ and $W=12-450 \mathrm{~km} ; M_{o} \sim W^{5.56}$ for SS events with $M_{w}=5.3-$ 7.8 and $W=4-30 \mathrm{~km} ; M_{o} \sim W^{2.27}$ for all slip-type events with $M_{w}=5.3-9.5$ and $W=4-240 \mathrm{~km}$. From a large number of all slip-type earthquakes with $M_{w}=5.4-9.2$, Thingbaijam et al. (2017) obtained $M_{o} \sim W^{3.45}[$ from $\log (W) \sim(0.435 \pm$ $0.005) M_{w}$ with $S D=0.087$ ] for RE events; $M_{o} \sim W^{4.64}$ [from $\log (W) \sim(0.323 \pm 0.047) M_{w}$ with $S D=0.099$ ] for NL events; $M_{o} \sim W^{5.75}$ [from $\log (W) \sim(0.261 \pm 0.026) M_{w}$ with $S D=0.105$ ] for SS events; and $M_{o} \sim W^{4.09}$ [from $\log (W) \sim(0.366 \pm 0.031)$ $M_{w}$ with $\left.S D=0.099\right]$ for subduction-interface events. They also gained $M_{o} \sim W^{7.14}$ for $M_{w} \leq 7.1$ and $M_{o} \sim W^{7.50}$ for $M_{w}>$ 7.1, suggesting a small difference in scaling relationships between $M_{o}$ and $W$ for the two ranges of $M_{o}$.

For SS and RE events with $M_{w}=5.6-7.8$ on low sliprate faults in New Zealand, Stirling et al. (2008) obtained a scaling relationship of $M_{w}$ in terms of both $L$ and $W: M_{w}$ $=4.18+(2 / 3) \log (W)+(4 / 3) \log (L)$. This relationship can simultaneously lead to $M_{o} \sim W^{1}$ and $M_{o} \sim L^{2}$.

The values of $n$ of $M_{o} \sim W^{n}$ are listed in Table 3, which cannot give us a substantial conclusion because the estimated values vary very much. This might also be due to a small number of data in some researches and high uncertainty of evaluating $W$. Based on the definition of $M_{o}$, the upper value of scaling exponent of $M_{o}-W$ is 3 , if the two scaling relationships: $D_{o} \sim W$ and $L \sim W$ exist simultaneously. However, numerous estimated values of the scaling exponent are larger than 3 . This is questionable and might be due to miss-estimates of $W$ from the aftershock area or from the finite-fault slip model.

\subsection{Modeling}

Based on the 1-D spring-slider model (denoted by the BK model) proposed by Burridge and Knopoff (1967) and its modified form, numerous studies have been made on the $M_{o}-L$ scaling relationship. The model and the details about the studies can be seen in Wang (1995a, b), and only some results are simply described below. Since the model consists of a series of sliders along the horizontal axis, there is no fault width, Carlson and Langer (1989) defined an earthquake moment $M_{o}{ }^{\prime}=\Sigma u_{i}$ to be the total displacement of a connected set of sliders, which slide during an event. This definition is different from $M_{o}$, and the definition of $M_{o}$ ' leads to $M_{o}{ }^{\prime}=N_{s} D_{o}$, where $N_{s}$ is the number of sliders slid during an event. This leads to $M_{o}{ }^{\prime} \sim L$, where $L$ is the rupture length of an event and is almost equal to $N a$, where " $a$ " is the spacing between two sliders in the equilibrium state as mentioned above. Hence, $M_{o}{ }^{\prime} \sim D_{o} L W \sim D_{o} A$, where the value of $W$ of the 1-D model is considered to be unity. This indicates that there is a positive correlation between $M_{o}$ and $M_{o}$ '.

Carlson et al. (1991) show that the simulated $M_{o}^{\prime}$ - $L$ distribution varies with the event size: $M_{o}{ }^{\prime} \sim L^{3 / 2}$ for small events and $M_{o}{ }^{\prime} \sim L$ for large ones. The simulated $M_{o}{ }^{\prime} \sim L$ distribution for intermediate-size events, cannot be interpreted by using a single power-law function. Wang (1995b) reported that only the rapidly velocity-weakening process can produce a well-defined power-law $M_{o}{ }^{\prime}-L$ relation. He also found that the degree of dissipation of energy cannot change this scaling law. His results do not show transition zone for intermediate-size events as pointed out by Carlson et al. (1991) and do give $M_{o}{ }^{\prime} \sim L^{2}$ for small events and of $M_{o}{ }^{\prime} \sim L$ for large ones. Based on continuum models associated with the 1-D BK model, several authors (e.g., Langer et al. 1996; Myers et al. 1996; Shaw 1997) found that $M_{o}{ }^{\prime} \sim L^{2}$ for the smallest events, and $M_{o}{ }^{\prime} \sim L$ for the largest ones. Meanwhile, there is a transition range, which cannot be described by a single power-law function, for the intermediate-sized events. Those simulated $M_{o} \sim L$ relations are obviously different from observed ones. The value of scaling exponent of simulated relationship is one smaller than that of observed relationship. This might be due to a fact that all simulations are made based on the 1-D model, while the observations come from natural 2-D fault zones. Hence, it should be significant to study further this problem using a 2-D BK model.

\section{APPLICATION OF SCALING LAWS TO SEISMIC HAZARD ASSESSMENT}

The application of scaling laws to assess seismic hazards and strong ground motions is an important and useful topic. Of course, it is necessary to select appropriate scaling laws for this purpose. Irikura and Miyake (2011) and Stirling et al. (2013) discussed the problem in details. Here, only a simple description is given because it is somehow out of scope of this review study. The present studies exhibit that scaling laws of source parameters of earthquake faults are regional dependent of different regional physical conditions of seismogenic zones. Meanwhile, regional scaling laws are often different from world-wide scaling laws inferred from global data. This would influence the assessment of seismic hazards in a region when the laws are lack in the region and must be taken from other regions. Hence, in order to assess seismic hazards in a region, it is necessary to use the scaling laws of source parameters inferred from the data of that region. On the other hand, in order to assess world-wild seismic hazards, for example the Global Earthquake Model (see https://www.globalquakemodel.org/), it is, of course, necessary to use the scaling laws of source parameters inferred from global data.

In addition, the quality of data and the selection of regression technique are also important on the inferred scaling laws. In the theory of probability, there is an important law, i.e., the so-called law of large numbers (cf. Bhattacharyya 


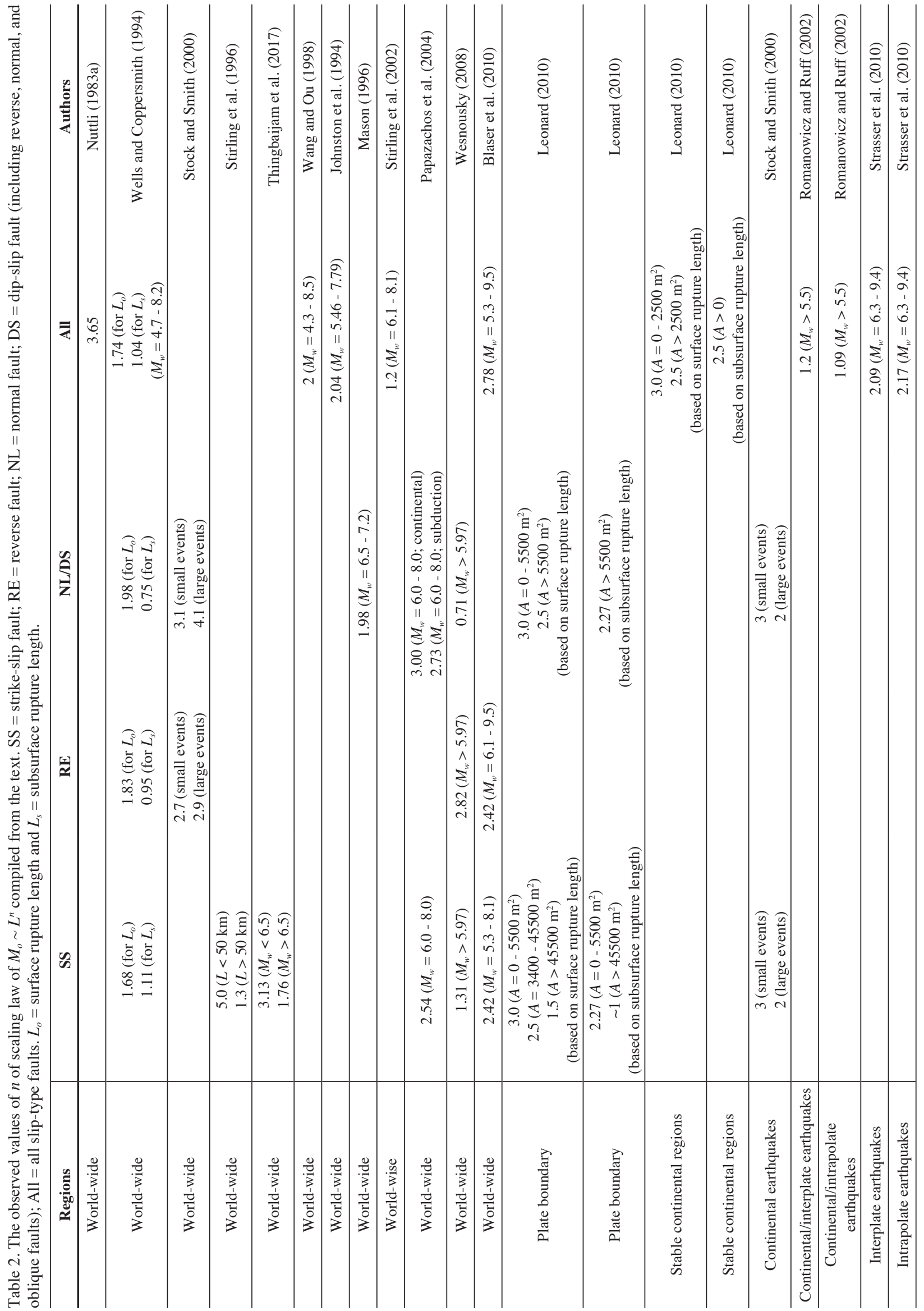







and Johnson 1977), which describes the result of conducting the same experiment a large number of times. Based on this law, the statistical results obtained from a large number of trials should be close to the expected value. Hence, the scaling laws inferred from a larger number of high-quality data could be more reliable than those from a smaller number of data. Meanwhile, the scaling laws inferred from the data by using the major-axis or orthogonal least-squared regression method would be more reliable than those by using the ordinary regression method for seismic hazard assessment.

\section{SUMMARY}

In this paper, the observational and theoretical studies of scaling of fault parameters, including static stress drop $(\Delta \sigma)$, the seismic moment $\left(M_{o}\right)$, the fault length $(L)$, fault width $(W)$, fault area $(A)$, average displacement $\left(D_{o}\right)$, and maximum displacement $\left(D_{\max }\right)$, are reviewed and main results are described below:

(1) Assumptions of constant $\Delta \sigma$, of earthquakes and selfsimilarity of faults: The assumption of constant $\Delta \sigma$ proposed by Aki (1967) is questionable.

(2) The $L$ - and $W$-models: According to given observed results, it is still difficult to determine which one is better than the other.

(3) $L-D_{o}$ and $L-D_{\max }$ relationships: Observations and theoretical results show nonlinear correlations between $L$ and $D_{o}$ as well as $D_{\max }$ and thus make the assumption of constant $\Delta \sigma$ questionable.

(4) Dependence of $D_{o}$ and $D_{\max }$ on $M_{o}$ : For all slip-type faults $M_{o}$ scales with $D_{o}$ almost in a form of $M_{o} \sim D_{o}^{2.67}$ and the scaling exponent is smaller for the dip-slip faults than for the strike-slip faults.

(5) $D_{o}-A$ scaling relationships: Given result essentially exhibits $D_{o} \sim A^{0.5}$.

(6) The scaling relationship between $M_{o}$ and the fault area, $A$, on $M_{o}$ : All observations confirm the scaling relationship of $M_{o} \sim A^{1.5}$.

(7) The scaling relationships between $W$ and $L$ : Given data have not yet lead to a confirmative correlation between the two source parameters.

(8) The scaling relationships between $M_{o}$ and $L$ as well as $W$ : For the $M_{o}-L$ scaling relationship, the values of $\mathrm{n}$ are larger for small earthquakes than for large events for all slip types, even though the estimated values are not exactly the same. This result is consistent with that inferred from the seismogenic zone. Since the respective estimated values of $\mathrm{n}$ for various slip types vary very much, no substantial conclusion can be made. For the $M_{o}-W$ scaling relationship, given data cannot lead to a substantial correlation between the two parameters and the estimated values of scaling exponent are unreasonably high.

(9) Modeling of $M_{o}-L$ scaling based on the one-dimensional spring-slider model in the presence of velocity-weaken- ing friction: The simulation $M_{o}{ }^{\prime} \sim L$ relations obtained by different authors are obviously different from observed ones. This might be due to a fact that simulations are made based on the 1-D model, while the observations come from natural 2-D fault zones.

(10) In order to assess seismic hazards in a region, it is necessary to use the scaling laws of source parameters inferred from the data of that region. On the other hand, in order to assess world-wild seismic hazards, it is necessary to use the scaling laws of source parameters inferred from global data.

Acknowledgements The author would like to thank Prof. Ruey-Juin Rau (Editor of Terrestrial, Atmospheric and Oceanic Sciences) and three anonymous reviewers for their valuable comments and suggestions to help him to substantially improve this article. This study was financially supported by Academia Sinica, the Ministry of Science and Technology (Grand No.: MOST 106-2116-M-001-005), and the Central Weather Bureau (Grand No.: MOTC-CWB105-E-02), Taiwan, ROC.

\section{REFERENCES}

Aki, K., 1966: Generation and propagation of $\mathrm{G}$ waves from the Niigata earthquake of June 16, 1964: Part 2. Estimation of earthquake moment, released energy, and stress-strain drop from the $\mathrm{G}$ wave spectrum. Bull. Earthquake Res. Inst., 44, 73-88.

Aki, K., 1967: Scaling law of seismic spectrum. J. Geophys. Res., 72, 1217-1231, doi: 10.1029/jz072i004p01217. [Link]

Aki, K., 1972: Scaling law of earthquake source time-function. Geophys. J. Int., 31, 3-25, doi: 10.1111/j.1365246x.1972.tb02356.x. [Link]

Aki, K., 1987: Magnitude-frequency relation for small earthquakes: A clue to the origin of $f_{\max }$ of large earthquakes. J. Geophys. Res., 92, 1349-1355, doi: 10.1029/ jb092ib02p01349. [Link]

Aki, K., 1992: Higher-order interrelations between seismogenic structures and earthquake processes. Tectonophysics, 211, 1-12, doi: 10.1016/0040-1951(92)90047a. [Link]

Aki, K. and P. G. Richards, 1980: Quantitative Seismology: Theory and Methods, W. H. Freeman and Co., San Francisco, 932 pp.

Allmann, B. P. and P. M. Shearer, 2009: Global variations of stress drop for moderate to large earthquakes. J. Geophys. Res., 114, B01310, doi: 10.1029/2008JB005821. [Link]

Anderson, J. G., S. G. Wesnousky, and M. W. Stirling, 1996: Earthquake size as a function of fault slip rate.

Bull. Seismol. Soc. Am., 86, 683-690.

Anderson, J. G., G. P. Biasi, and S. G. Wesnousky, 2017: 
Fault-scaling relationships depend on the average faultslip rate. Bull. Seismol. Soc. Am., 107, 2561-2577, doi: 10.1785/0120160361. [Link]

Archuleta, R. J., 1982: Analysis of near-source static and dynamic measurements from the 1979 Imperial Valley earthquake. Bull. Seismol. Soc. Am., 72, 1927-1956.

Asano, K. and T. Iwata, 2011: Characterization of stress drops on asperities estimated from the heterogeneous kinematic slip model for strong motion prediction for inland crustal earthquakes in Japan. Pure Appl. Geophys., 168, 105-116, doi: 10.1007/s00024-010-0116-y. [Link]

Asano, K., T. Iwata, and K. Irikura, 2003: Source characteristics of shallow intraslab earthquakes derived from strong-motion simulations. Earth Planets Space, 55, e5-e8, doi: 10.1186/bf03351744. [Link]

Bhattacharyya, G. K. and R. A. Johnson, 1977: Statistical Concepts and Methods, Wiley, $656 \mathrm{pp}$.

Bilek, S. L. and T. Lay, 1999: Rigidity variations with depth along interplate megathrust faults in subduction zones. Nature, 400, 443-446, doi: 10.1038/22739. [Link]

Bizzarri, A., 2011: On the deterministic description of earthquakes. Rev. Geophys., 49, RG3002, doi: 10.1029/2011RG000356. [Link]

Blaser, L., F. Krüger, M. Ohrnberger, and F. Scherbaum, 2010: Scaling relations of earthquake source parameter estimates with special focus on subduction environment. Bull. Seismol. Soc. Am., 100, 2914-2926, doi: 10.1785/0120100111. [Link]

Bodin, P. and J. N. Brune, 1996: On the scaling of slip with rupture length for shallow strike-slip earthquakes: Quasi-static models and dynamic rupture propagation. Bull. Seismol. Soc. Am., 86, 1292-1299.

Boore, D. M. and G. M. Atkinson, 1987: Stochastic prediction of ground motion and spectral response parameters at hard-rock sites in eastern North America. Bull. Seismol. Soc. Am., 77, 440-467.

Bracewell, R. N., 1986: The Fourier Transform and Its Applications, McGraw-Hill, New York, 474 pp.

Burridge, R. and L. Knopoff, 1967: Model and theoretical seismicity. Bull. Seismol. Soc. Am., 57, 341-371.

Carlson, J. M. and J. S. Langer, 1989: Mechanical model of an earthquake fault. Phys. Rev. A, 40, 6470-6484, doi: 10.1103/physreva.40.6470. [Link]

Carlson, J. M., J. S. Langer, B. E. Shaw, and C. Tang, 1991: Intrinsic properties of a Burridge-Knopoff model of an earthquake fault. Phys. Rev. A, 44, 884-897, doi: 10.1103/physreva.44.884. [Link]

Carpenter, N. S., S. J. Payne, and A. L. Schafer, 2012: Toward reconciling magnitude discrepancies estimated from paleoearthquake data. Seismol. Res. Lett., 83, 555-565, doi: 10.1785/gssrl.83.3.555. [Link]

Chinnery, M. A., 1964: The strength of the Earth's crust under horizontal shear stress. J. Geophys. Res., 69, 2085-
2089, doi: 10.1029/jz069i010p02085. [Link]

Das, S., 1982: Appropriate boundary conditions for modeling very long earthquakes and physical consequences. Bull. Seismol. Soc. Am., 72, 1911-1926.

Denolle, M. A. and P. M. Shearer, 2016: New perspectives on self-similarity for shallow thrust earthquakes. J. Geophys. Res., 121, 6533-6565, doi: 10.1002/2016JB013105. [Link]

Dietz, L. D. and W. L. Ellsworth, 1990: The October 17, 1989, Loma Prieta, California, Earthquake and its aftershocks: Geometry of the sequence from high-resolution locations. Geophys. Res. Lett., 17, 1417-1420, doi: 10.1029/GL017i009p01417. [Link]

Dowrick, D. J. and D. A. Rhoades, 2004: Relations between earthquake magnitude and fault rupture dimensions: How regionally variable are they? Bull. Seismol. Soc. Am., 94, 776-788, doi: 10.1785/0120030151. [Link]

Fletcher, J. B., 1982: A comparison between the tectonic stress measured in situ and stress parameters from induced seismicity at Monticello Reservoir, South Carolina. J. Geophys. Res., 87, 6931-6944, doi: 10.1029/ jb087ib08p06931. [Link]

Fletcher, J. B., L. C. Haar, F. L. Vernon, J. N. Brune, T. C. Hanks, and J. Berger, 1986: The effects of attenuation on the scaling of source parameters for earthquakes at Anza, California. In: Das, S., J. Boatwright, and C. H. Scholz (Eds.), Earthquake Source Mechanics, Geophysical Monograph Series, Vol. 37, American Geophysical Union, 331-338, doi: 10.1029/GM037p0331. [Link]

Funning, G., A. M. Ferreira, J. M. Weston, and H. Bloomfield, 2013: Investigating earthquake self-similarity using a 20 year catalog of source parameters derived from InSAR data. American Geophysical Union, Fall Meeting 2013, NG11A-1587.

Geller, R. J., 1976: Scaling relations for earthquake source parameters and magnitudes. Bull. Seismol. Soc. Am., 66, 1501-1523.

Graves, R., T. H. Jordan, S. Callaghan, E. Deelman, E. Field, G. Juve, C. Kesselman, P. Maechling, G. Mehta, K. Milner, D. Okaya, P. Small, and K. Vahi, 2011: CyberShake: A physics-based seismic hazard model for southern California. Pure Appl. Geophys., 168, 367381, doi: 10.1007/s00024-010-0161-6. [Link]

Gutenberg, B. and C. F. Richter, 1956: Magnitude and energy of earthquakes. Ann. Geophys., 9, 1-15, doi: 10.4401/ag-5590. [Link]

Haar, L. C., J. B. Fletcher, and C. S. Mueller, 1984: The 1982 Enola, Arkansas, swarm and scaling of ground motion in the eastern United States. Bull. Seismol.Soc. Am., 74, 2463-2482.

Hanks, T. C., 1977: Earthquake stress drops, ambient tectonic stresses and stresses that drive plate motions. Pure Appl. Geophys., 115, 441-458, doi: 10.1007/ BF01637120. [Link] 
Hanks, T. C. and W. H. Bakun, 2002: A bilinear sourcescaling model for M-log A observations of continental earthquakes. Bull. Seismol. Soc. Am., 92, 1841-1846, doi: 10.1785/0120010148. [Link]

Hanks, T. C. and W. H. Bakun, 2008: M-logA observations for recent large earthquakes. Bull. Seismol. Soc. Am., 98, 490-494, doi: 10.1785/0120070174. [Link]

Hanks, T. C. and H. Kanamori, 1979: A moment magnitude scale. J. Geophys. Res., 84, 2348-2350, doi: 10.1029/ JB084iB05p02348. [Link]

Hardebeck, J. L. and A. Aron, 2009: Earthquake stress drops and inferred fault strength on the Hayward fault, east San Francisco Bay, California. Bull. Seismol. Soc. Am., 99, 1801-1814, doi: 10.1785/0120080242. [Link]

Hartzell, S. H. and T. H. Heaton, 1988: Failure of self-similarity for large $\left(M_{\omega}>81 / 4\right)$ earthquakes. Bull. Seismol. Soc. Am., 78, 478-488.

Heimpel, M. and P. Malin, 1998: Aseismic slip in earthquake nucleation and self-similarity: Evidence from Parkfield, California. Earth Planet. Sci. Lett., 157, 249254, doi: 10.1016/s0012-821x(98)00035-1. [Link]

Hillers, G. and S. G. Wesnousky, 2008: Scaling relations of strike-slip earthquakes with different slip-rate-dependent properties at depth. Bull. Seismol. Soc. Am., 98, 1085-1101, doi: 10.1785/0120070200. [Link]

Huang, W. G., J. H. Wang, B. S. Huang, K. C. Chen, T. M. Chang, R. D. Hwang, H. C. Chiu, and C. C. P. Tsai, 2001: Estimates of source parameters for the 1999 Chi-Chi, Taiwan, earthquake based on Brune's source model. Bull. Seismol. Soc. Am., 91, 1190-1198.

Hwang, R. D., J. H. Wang, B. S. Huang, K. C. Chen, W. G. Huang, T. M. Chang, H. C. Chiu, and C. C. P. Tsai, 2001: Estimates of stress drop of the Chi-Chi, Taiwan, earthquake of 20 September 1999 from near-field seismograms. Bull. Seismol. Soc. Am., 91, 1158-1166.

Ichinose, G. A., H. K. Thio, and P. G. Somerville, 2006: Moment tensor and rupture model for the 1949 Olympia, Washington, earthquake and scaling relations for cascadia and global intraslab earthquakes. Bull. Seismol. Soc. Am., 96, 1029-1037, doi: 10.1785/0120050132. [Link]

Imanishi, K. and W. L. Ellsworth, 2006: Source scaling relationships of microearthquakes at Parkfield, CA, determined using the SAFOD pilot hole seismic array. In: Abercrombie, R. E., A. McGarr, G. Di Toro, and H. Kanamori (Eds.), Earthquakes: Radiated Energy and the Physics of Faulting, Vol. 170, Geophysical Monograph Series, American Geophysical Union, Washington, D.C., 81-90, doi: 10.1029/170GM10. [Link]

Irikura, K. and H. Miyake, 2011: Recipe for Predicting Strong Ground Motion from Crustal Earthquake Scenarios. Pure Appl.Geophys., 168, 85-104, doi: 10.1007/ s00024-010-0150-9. [Link]

Johnston, A. C., L. R. Kanter, K. J. Coppersmith, and C.
A. Cornell, 1994: Seismotectonic interpretations and conclusions from the stable continental region seismicity database. The Earthquakes of Stable Continental Regions, Vol. 1: Assessment of Large Earthquake Potential, Electric Power Research Institute, Palo Alto, California.

Kanamori, H. and C. R. Allen, 1986: Earthquake repeat time and average stress drop. In: Das, S., J. Boatwright, and C. H. Scholz (Eds.), Earthquake Source Mechanics, Vol. 37, Geophysical Monograph Series, American Geophysical Union, 227-235, doi: 10.1029/ GM037p0227. [Link]

Kanamori, H. and D. L. Anderson, 1975: Theoretical basis of some empirical relations in seismology. Bull. Seismol. Soc. Am., 65, 1073-1095.

Kanamori, H., J. Mori, and T. H. Heaton, 1990: The 3 December 1988, Pasadena earthquake $\left(\mathrm{M}_{\mathrm{L}}=4.9\right)$ recorded with the very broadband system in Pasadena. Bull. Seismol. Soc. Am., 80, 483-487.

Kanamori, H., J. Mori, E. Hauksson, T. H. Heaton, L. K. Hutton, and L. M. Jones, 1993: Determination of earthquake energy release and $M_{L}$ using TERRAscope. Bull. Seismol. Soc. Am., 83, 330-346.

Kase, Y., 2010: Slip-length scaling law for strike-slip multiple segment earthquakes based on dynamic rupture simulations. Bull. Seismol. Soc. Am., 100, 473-481, doi: 10.1785/0120090090. [Link]

King, G. C. P. and S. G. Wesnousky, 2007: Scaling of fault parameters for continental strike-slip earthquakes. Bull. Seismol. Soc. Am., 97, 1833-1840, doi: 10.1785/0120070048. [Link]

Knopoff, L., 1958: Energy release in earthquakes. Geophys. J. Int., 1, 44-52, doi: 10.1111/j.1365-246X.1958. tb00033.x. [Link]

Langer, J. S., J. M. Carlson, C. R. Myers, and B. E. Shaw, 1996: Slip complexity in dynamic models of earthquake faults. Proc. Natl. Acad. Sci. USA, 93, 3825-3829.

Leonard, M., 2010: Earthquake fault scaling: Self-consistent relating of rupture length, width, average displacement, and moment release. Bull. Seismol. Soc. Am., 100, 1971-1988, doi: 10.1785/0120090189. [Link]

Leonard, M., 2014: Self-consistent earthquake fault-scaling relations: Update and extension to stable continental strike-slip faults. Bull. Seismol. Soc. Am., 104, 29532965, doi: 10.1785/0120140087. [Link]

Liu-Zeng, J., T. Heaton, and C. DiCaprio, 2005: The effect of slip variability on earthquake slip-length scaling. Geophys. J. Int., 162, 841-849, doi: 10.1111/j.1365246x.2005.02679.x. [Link]

Luo, Y., J. P. Ampuero, K. Miyakoshi, and K. Irikura, 2017: Surface rupture effects on earthquake moment-area scaling relations. Pure Appl. Geophys., 174, 33313342, doi: 10.1007/s00024-017-1467-4. [Link]

Ma, K.-F. and H. Kanamori, 1994: Broadband waveform 
observation of the 28 June 1991 Sierra Madre earthquake sequence $\left(M_{L}=5.8\right)$. Bull. Seismol. Soc. Am., 84, 1725-1738.

Mai, P. M. and G. C. Beroza, 2000: Source scaling properties from finite-fault-rupture models. Bull. Seismol. Soc. Am., 90, 604-615, doi: 10.1785/0119990126. [Link]

Manighetti, I., M. Campillo, C. Sammis, P. M. Mai, and G. King, 2005: Evidence for self-similar, triangular slip distributions on earthquakes: Implications for earthquake and fault mechanics. J. Geophys. Res., 110, B05302, doi: 10.1029/2004JB003174. [Link]

Manighetti, I., M. Campillo, S. Bouley, and F. Cotton, 2007: Earthquake scaling, fault segmentation, and structural maturity. Earth Planet. Sci. Lett., 253, 429-438, doi: 10.1016/j.eps1.2006.11.004. [Link]

Mason, D. B., 1996: Earthquake magnitude potential of the Intermountain Seismic Belt, USA, from surface-parameter scaling of late Quaternary faults.Bull. Seismol. Soc. Am., 86, 1487-1506.

Murotani, S., H. Miyake, and K. Koketsu, 2008: Scaling of characterized slip models for plate-boundary earthquakes. Earth Planets Space, 60, 987-991, doi: 10.1186/bf03352855. [Link]

Murotani, S., K. Satake, and Y. Fujii, 2013: Scaling relations of seismic moment, rupture area, average slip, and asperity size for $\mathrm{M} \sim 9$ subduction-zone earthquakes. Geophys. Res. Lett., 40, 5070-5074, doi: 10.1002/ grl.50976. [Link]

Myers, C. R., B. E. Shaw, and J. S. Langer, 1996: Slip complexity in a crustal-plane model of an earthquake fault. Phys. Rev. Lett., 77, 972-975, doi: 10.1103/physrevlett.77.972. [Link]

Nadeau, R. M. and L. R. Johnson, 1998: Seismological studies at Parkfield VI: Moment release rates and estimates of source parameters for small repeating earthquakes. Bull. Seismol. Soc. Am., 88, 790-814.

Nuttli, O. W., 1983a: Average seismic source-parameter relations for mid-plate earthquakes. Bull. Seismol. Soc. Am., 73, 519-535.

Nuttli, O. W., 1983b: Empirical magnitude and spectral scaling relations for mid-plate and plate-margin earthquakes. Tectonophysics, 93, 207-223, doi: 10.1016/0040-1951(83)90278-0. [Link]

Papazachos, B. C., E. M. Scordilis, D. G. Panagiotopoulos, C. B. Papazachos, and G. F. Karakaisis, 2004: Global relations between seismic fault parameters and moment magnitude of earthquakes. Bulletin of the Geological Society of Greece, 36, 1482-1489, doi: 10.12681/ bgsg.16538. [Link]

Patanè, D., F. Ferrucci, E. Giampiccolo, and L. Scaramuzzino, 1997: Source scaling of microearthquakes at Mt. Etna volcano and in the Calabrian Arc (southern Italy). Geophys. Res. Lett., 24, 1879-1882, doi: 10.1029/97gl01745. [Link]
Pegler, G. and S. Das, 1996: Analysis of the relationship between seismic moment and fault length for large crustal strike-slip earthquakes between 1977-92. Geophys. Res. Lett., 23, 905-908, doi: 10.1029/96gl00963. [Link]

Prieto, G. A., P. M. Shearer, F. L. Vernon, and D. Kilb, 2004: Earthquake source scaling and self-similarity estimation from stacking $\mathrm{P}$ and $\mathrm{S}$ spectra. J. Geophys. Res., 109, B08310, doi: 10.1029/2004jb003084. [Link]

Romanowicz, B., 1992: Strike-slip earthquakes on quasivertical transcurrent faults: Inferences for general scaling relations. Geophys. Res. Lett., 19, 481-484, doi: 10.1029/92g100265. [Link]

Romanowicz, B. and L. J. Ruff, 2002: On moment-length scaling of large strike slip earthquakes and the strength of faults. Geophys. Res. Lett., 29, 45-1-45-4, doi: 10.1029/2001gl014479. [Link]

Romanowicz, B. and J. B. Rundle, 1993: On scaling relations for large earthquakes. Bull. Seismol. Soc. Am., 83, 1294-1297.

Scholz, C. H., 1982: Scaling laws for large earthquakes: Consequences for physical models. Bull. Seismol. Soc. Am., 72, 1-14.

Scholz, C. H., 1990: The Mechanics of Earthquakes and Faulting, Cambridge University Press, Cambridge, $461 \mathrm{pp}$.

Scholz, C. H., 1994a: A reappraisal of large earthquake scaling. Bull. Seismol. Soc. Am., 84, 215-218.

Scholz, C. H., 1994b: Reply to comments on "A reappraisal of large earthquake scaling” by C. Scholz. Bull. Seismol. Soc. Am., 84, 1677-1678.

Scholz, C. H., 1997: Size distributions for large and small earthquakes. Bull. Seismol. Soc. Am., 87, 1074-1077.

Scholz, C. H. and P. A. Cowie, 1990: Determination of total strain from faulting using slip measurements. Nature, 346, 837-839, doi: 10.1038/346837a0. [Link]

Scholz, C. H., C. A. Aviles, and S. G. Wesnousky, 1986: Scaling differences between large interplate and intraplate earthquakes. Bull. Seismol. Soc. Am., 76, 65-70.

Schwartz, D. P. and K. J. Coppersmith, 1984: Fault behavior and characteristic earthquakes: Examples from the Wasatch and San Andreas Fault Zones. J. Geophys. Res., 89, 5681-5698, doi: 10.1029/jb089ib07p05681. [Link]

Shaw, B. E., 1997: Model quakes in the two-dimensional wave equation. J. Geophys. Res., 102, 27367-27377, doi: 10.1029/97jb02786. [Link]

Shaw, B. E., 2009: Constant stress drop from small to great earthquakes in magnitude-area scaling. Bull. Seismol. Soc. Am., 99, 871-875, doi: 10.1785/0120080006. [Link]

Shaw, B. E. and C. H. Scholz, 2001: Slip-length scaling in large earthquakes: Observations and theory and implications for earthquake physics. Geophys. Res. Lett., 28, 
2995-2998 , doi: 10.1029/2000g1012762. [Link]

Shaw, B. E. and S. G. Wesnousky, 2008: Slip-length scaling in large earthquakes: The role of deep-penetrating slip below the seismogenic layer. Bull. Seismol. Soc. Am., 98, 1633-1641, doi: 10.1785/0120070191. [Link]

Shimazaki, K., 1986: Small and large earthquakes: The effects of the thickness of seismogenic layer and the free surface. In: Das, S., J. Boatwright, and C. H. Scholz (Eds.), Earthquake Source Mechanics, Vol. 37, American Geophysical Union, 209-216, doi: 10.1029/ GM037p0209. [Link]

Sibson, R. H., 1982: Fault zone models, heat flow, and the depth distribution of earthquakes in the continental crust of the United States. Bull. Seismol. Soc. Am., 72, 151-163.

Sibson, R. H., 1983: Continental fault structure and the shallow earthquake source. J. Geol. Soc., 140, 741-767, doi: 10.1144/gsjgs.140.5.0741. [Link]

Sibson, R. H., 1984: Roughness at the base of the seismogenic zone: Contributing factors. J. Geophys. Res., 89, 5791-5799, doi: 10.1029/jb089ib07p05791. [Link]

Sibson, R. H., 1986: Earthquakes and rock deformation in crustal zones. Апnи. Rev. Earth Planet. Sci., 14, 149175, doi: 10.1146/annurev.earth.14.1.149. [Link]

Skarlatoudis, A. A., P. G. Somerville, and H. K. Thio, 2016: Source-scaling relations of interface subduction earthquakes for strong ground motion and tsunami simulation. Bull. Seismol. Soc. Am., 106, 1652-1662, doi: 10.1785/0120150320. [Link]

Somerville, P. G., J. P. McLaren, L. V. LeFevre, R. W. Burger, and D. V. Helmberger, 1987: Comparison of source scaling relations of eastern and western North American earthquakes. Bull. Seismol. Soc. Am., 77, 322-346.

Somerville, P. G., K. Irikura, R. Graves, S. Sawada, D. Wald, N. Abrahamson, Y. Iwasaki, T. Kagawa, N. Smith, and A. Kowada, 1999: Characterizing crustal earthquake slip models for the prediction of strong ground motion. Seismol. Res. Lett., 70, 59-80, doi: 10.1785/gssrl.70.1.59. [Link]

Somerville, P. G., N. Collins, and R. Graves, 2006: Magnitude-rupture area scaling of large strike-slip earthquakes. Final Technical Report Award No. 05-HQGR-0004, USGS.

Starr, A. T., 1928: Slip in a crystal and rupture in a solid due to shear. Math. Proc. Camb. Phil. Soc., 24, 489-500, doi: 10.1017/s0305004100014626. [Link]

Stirling, M. W. and J. G. Anderson, 2018: Magnitude as a function of rupture length and slip rate for recent large New Zealand earthquakes. Bull. Seismol. Soc. Am., 108, 1623-1629, doi: 10.1785/0120170284. [Link]

Stirling, M. W., S. G. Wesnousky, and K. Shimazaki, 1996: Fault trace complexity, cumulative slip, and the shape of the magnitude-frequency distribution for strike-slip faults: A global survey. Geophys. J.Int., 124, 833-868, doi: 10.1111/j.1365-246X.1996.tb05641.x. [Link]

Stirling, M. W., D. Rhoades, and K. Berryman, 2002: Comparison of earthquake scaling relations derived from data of the instrumental and preinstrumental era. Bull. Seismol. Soc. Am., 92, 812-830, doi: 10.1785/0120000221. [Link]

Stirling, M. W., M. C. Gerstenberger, N. J. Litchfield, G. H. McVerry, W. D. Smith, J. Pettinga, and P. Barnes, 2008: Seismic hazard of the Canterbury region, New Zealand: New earthquake source model and methodology. Bull. New Zeal. Soc. Earthquake Eng., 41, 51-67.

Stirling, M. W., T. Goded, K. Berryman, and N. Litchfield, 2013: Selection of earthquake scaling relationships for seismic-hazard analysis. Bull. Seismol. Soc. Am., 103, 2993-3011, doi: 10.1785/0120130052. [Link]

Stock, C. and E. G. C. Smith, 2000: Evidence for different scaling of earthquake source parameters for large earthquakes depending on faulting mechanism. Geophys. J. Int., 143, 157-162, doi: 10.1046/j.1365246x.2000.00225.x. [Link]

Strasser, F. O., M. C. Arango, and J. J. Bommer, 2010: Scaling of the source dimensions of interface and intraslab subduction-zone earthquakes with moment magnitude. Seismol. Res. Lett., 81, 941-950, doi: 10.1785/ gssrl.81.6.941. [Link]

Thingbaijam, K. K. S. and P. M. Mai, 2016: Evidence for truncated exponential probability distribution of earthquake slip. Bull. Seismol. Soc. Am., 106, 1802-1816, doi: 10.1785/0120150291. [Link]

Thingbaijam, K. K. S., P. Martin Mai, and K. Goda, 2017: New empirical earthquake source-scaling laws. Bull. Seismol. Soc. Am., 107, 2225-2246, doi: 10.1785/0120170017. [Link]

Tsuboi, C., 1956: Earthquake energy, earthquake volume, aftershock area, and strength of the earth's crust. J. Phys. Earth, 4, 63-66, doi; 10.4294/jpe1952.4.63. [Link]

Villamor, P., K. R. Berryman, T. Webb, M. Stirling, P. McGinty, G. Downes, J. Harris, and N. Litchfield, 2001: Waikato seismic loads: Revision of seismic source characterisation. GNS Client Report 2001/59.

Wang, J.-H., 1995a: Effect of seismic coupling on the scaling of seismicity. Geophys. J. Int., 121, 475-488, doi: 10.1111/j.1365-246x.1995.tb05727.x. [Link]

Wang, J.-H., 1995b: A study of rupture length vs. moment for synthetic earthquakes. Pure Appl. Geophys., 144, 211-228, doi: 10.1007/bf00878632. [Link]

Wang, J.-H., 1997: On the frequency distribution of rupture lengths of earthquakes synthesized from a one-dimensional dynamical lattice model. J. Phys. Earth, 45, 363-381, doi: 10.4294/jpe1952.45.363. [Link]

Wang, J.-H., 2015: The energy-magnitude scaling law for $M_{s} \leq 5.5$ earthquakes. J. Seismol., 19, 647-652, doi: 10.1007/s10950-014-9473-9. [Link] 
Wang, J.-H. and S.-S. Ou, 1998: On scaling of earthquake faults. Bull. Seismol. Soc. Am., 88, 758-766.

Wells, D. L. and K. J. Coppersmith, 1994: New empirical relationships among magnitude, rupture length, rupture width, rupture area, and surface displacement. Bull. Seismol. Soc. Am., 84, 974-1002.

Wen, Y. Y., S. Y. Chao, Y. T. Yen, and S. Wen, 2017: Source characteristics of moderate-to-strong earthquakes in the Nantou area, Taiwan: Insight from strong ground motion simulations. Earth Planets Space, 69, doi: 10.1186/s40623-017-0720-5. [Link]

Wesnousky, S. G., 2006: Predicting the endpoints of earthquake ruptures. Nature, 444, 358-360, doi: 10.1038/ nature05275. [Link]

Wesnousky, S. G., 2008: Displacement and geometrical characteristics of earthquake surface ruptures: Issues and implications for seismic-hazard analysis and the process of earthquake rupture. Bull. Seismol. Soc. Am., 98, 1609-1632, doi: 10.1785/0120070111. [Link]
Wesnousky, S. G. and G. P. Biasi, 2011: The length to which an earthquake will go to rupture. Bull. Seismol. Soc. Am., 101, 1948-1950, doi: 10.1785/0120110013. [Link]

WGCEP (Working Group on California Earthquake Probabilities), 2008: The Uniform California Earthquake Rupture Forecast, version 2 (UCERF 2). USGS Numbered Series, Open-File Report 2007-1437, Geological Survey (U.S.), doi: 10.3133/ofr20071437. [Link]

Wyss, M., 1979: Estimating maximum expectable magnitude of earthquakes from fault dimensions. Geology, 7, 336-340, doi: 10.1130/0091-7613(1979)7<336:EM EMOE $>2.0 . \mathrm{CO} ; 2$. [Link]

Yen, Y.-T. and K.-F. Ma, 2011: Source-scaling relationship for M 4.6-8.9 earthquakes, specifically for earthquakes in the collision zone of Taiwan. Bull. Seismol. Soc. Am., 101, 464-481, doi: 10.1785/0120100046. [Link]

York, D., 1967: The best isochron. Earth Planet. Sci.Lett., 2, 479-482, doi: 10.1016/0012-821x(67)90193-8. [Link] 